\title{
Homogenization of time-harmonic Maxwell's equations in nonhomogeneous plasmonic structures
}

\author{
Matthias Maier ${ }^{\mathrm{a}}$, Dionisios Margetis $^{\mathrm{b}}$, Antoine Mellet $^{\mathrm{c}}$ \\ ${ }^{a}$ Department of Mathematics, Texas A\&M University, 3368 TAMU, College Station, TX \\ 77843, USA \\ ${ }^{b}$ Department of Mathematics, and Institute for Physical Science and Technology, and \\ Center for Scientific Computation and Mathematical Modeling, University of Maryland, \\ College Park, Maryland 20742, USA \\ ${ }^{c}$ Department of Mathematics, and Center for Scientific Computation and Mathematical \\ Modeling, University of Maryland, College Park, Maryland 20742, USA
}

\begin{abstract}
We carry out the homogenization of time-harmonic Maxwell's equations in a periodic, layered structure made of two-dimensional (2D) metallic sheets immersed in a heterogeneous and in principle anisotropic dielectric medium. In this setting, the tangential magnetic field exhibits a jump across each sheet. Our goal is the rigorous derivation of the effective dielectric permittivity of the system from the solution of a local cell problem via suitable averages. Each sheet has a fine-scale, inhomogeneous and possibly anisotropic surface conductivity that scales linearly with the microstructure scale, $d$. Starting with the weak formulation of the requisite boundary value problem, we prove the convergence of its solution to a homogenization limit as $d$ approaches zero. The effective permittivity and cell problem express a bulk average from the host dielectric and a surface average germane to the 2D material (metallic layer). We discuss implications of this analysis in the modeling of plasmonic crystals.
\end{abstract}

Keywords: Homogenization, Two-scale convergence, Time-harmonic Maxwell's equations, Layered structures, Jump condition on hypersurface; AMS Subject Classification: 35B27, 35Q60, 74Q10, 78A40

\section{Introduction}

Recent advances in the design and synthesis of thin materials have challenged traditional notions of optics such as the diffraction limit. The emerging class of metamaterials enable the control of the path and dispersion of light, which may in turn result in unusual optical phenomena that include no refraction ("epsilon near zero" effect) and negative refraction [18, 21 24, 34, 37]. In fact, the

Email addresses: maier@math.tamu.edu (Matthias Maier), dio@math.umd.edu (Dionisios Margetis), mellet@math.umd.edu (Antoine Mellet) 
optical conductivity of certain two-dimensional (2D) materials in the infrared spectrum permits the excitation of short-scale electromagnetic surface waves, called surface plasmon-polaritons, in the electron plasma under the appropriate polarization of the incident field [7, 13, 19, 31]. This type of wave is tightly confined near the 2D material. The existence of this wave has inspired the design of layered plasmonic structures that exhibit unconventional optical properties via the tuning of frequency or geometry [13, 21 23]. This physical prospect motivates our present work.

In a layered structure of $2 \mathrm{D}$ metallic sheets, the surface plasmon-polaritons excited in the electron plasma of the layers may constructively interfere in the dielectric host. This wave coupling can be enhanced for small enough interlayer spacing at the microscale; and can give rise to a slowly varying wave that propagates through the structure at the macroscale. By a suitable adjustment of the operating frequency or interlayer spacing, this wave may experience no phase delay [21, 23]. Mathematically, it is tempting to view this possibility as an outcome of homogenization, expecting that there is an effective description of wave propagation as the spacing approaches zero. The phase of the optical conductivity of each sheet plays a key role.

In this paper, we rigorously carry out the homogenization of a boundary value problem for the time harmonic Maxwell equations in a periodic, layered structure. The geometry consists of $2 \mathrm{D}$ plasmonic sheets in a heterogeneous dielectric medium. The surface conductivity, $\sigma^{d}$, of each sheet varies spatially with the microstructure scale $d$ and may be anisotropic. The dielectric permittivity, $\varepsilon^{d}$, of the host medium has an analogous, $d$-periodic microstructure in the ambient space and can be anisotropic. Our main result is the rigorous extraction of an effective dielectric permittivity and the related cell problem as $d \rightarrow 0$. Specifically, we complete the following main tasks.

- We develop the weak formulation for the associated boundary value problem of Maxwell's equations for the electromagnetic field $\left(\boldsymbol{E}^{d}, \boldsymbol{H}^{d}\right)$ in some generality. The tangential vector component, $\boldsymbol{H}_{T}^{d}$, of the magnetic field obeys a jump condition on each sheet; and the jump is proportional to $\sigma^{d} \boldsymbol{E}_{T}^{d}$, the current induced on the sheet. We make the assumption that $\sigma^{d}$ scales linearly with $d$, which is consistent with the experimentally observed fine-scale surface plasmon-polaritons.

- We address the simplified case with planar sheets, and scalar $\varepsilon^{d}$ and $\sigma^{d}$ first. In this vein, we prove a theorem (Theorem 2.3) asserting that for fixed $d$, the weak formulation admits a unique solution in an appropriate function space.

- We then show that the electromagnetic field $\left(\boldsymbol{E}^{d}, \boldsymbol{H}^{d}\right)$ converges weakly in $L^{2}$ to the solution $(\mathcal{E}, \mathcal{H})$ of the homogenized problem (Theorem 2.4). The homogenization limit reveals the effective permittivity, $\varepsilon^{\text {eff }}$, via a suitable average and the solution of a local cell problem; cf. (4). To obtain these results, we establish requisite a priori estimates in the context of two-scale 
convergence. For an overview of important results related to two-scale convergence, see Appendix A.

- We discuss the relevance of our model and analysis to the application area of plasmonics, especially the design of plasmonic crystals that exhibit no refraction (epsilon-near-zero effect).

- We point out extensions of our analysis to more general settings. In particular, our analysis can treat tensorial parameters $\varepsilon^{d}$ and $\sigma^{d}$, and non-planar sheets (see Appendix B).

In our analysis, for the sake of mathematical convenience we assume that the bulk material surrounding the metallic sheets is slightly lossy. This assumption, which is not uncommon in electromagnetics [26], amounts to the addition of a small, positive imaginary part to the dielectric permittivity $\varepsilon^{d}$ (under an $e^{-i \omega t}$ time dependence). Consequently, we conveniently obtain the desired a priori estimates for $\left(\boldsymbol{E}^{d}, \boldsymbol{H}^{d}\right)$.

There is extensive literature in the theory of periodic homogenization that is akin to our approach; see, e.g., [1, 3- 6, 11, 29, 32, 38-40]. Notably, the idea underlying the two-scale asymptotic analysis for $\left(\boldsymbol{E}^{d}, \boldsymbol{H}^{d}\right)$ can be found in [6, 30]; and our proof of homogenization relies on the known notion of twoscale convergence [1, 29]. In the setting of time-harmonic Maxwell's equations, our analysis brings forth the feature of averaging on hypersurfaces (metallic sheets) across which the magnetic field undergoes a jump involving the surface conductivity, $\sigma^{d}$. A similar jump condition is considered in [4], albeit in a different geometric setting which is motivated by geophysical applications: In [4] the jump condition accounts for interfacial currents that are present along the closed surfaces that separate two distinct phases of a composite material (with periodic structure). In our setting, on the other hand, surface currents on large $2 D$ sheets are discussed. This suggests a different approach to handle the contribution of these currents in our proof than the one employed in [4]. We refer to Sections 1.4 and 5 for further discussion and a comparison of the two problems and respective approaches.

We focus on the rigorous analysis of the periodic homogenization for plasmonic layered structures. Hence, numerical computations tailored to applications lie beyond our present scope, and will be the subject of future work. We assume that the reader is familiar with the fundamentals of classical electromagnetic wave theory; for extensive treatments of this subject, see, e.g., [26, 33]. The $e^{-i \omega t}$ time dependence is employed throughout.

\subsection{Problem formulation}

Our goal with this work is to extract effective material parameters in timeharmonic Maxwell's equations for layered systems of stacked, metallic sheets immersed in a non-homogeneous medium.

For ease of discussion, we will introduce the homogenization problem in this section for the (infinite) domain $\mathbb{R}^{3}$, assuming suitable boundary conditions. 


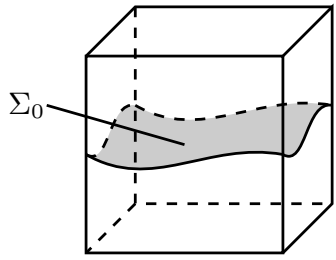

(a)

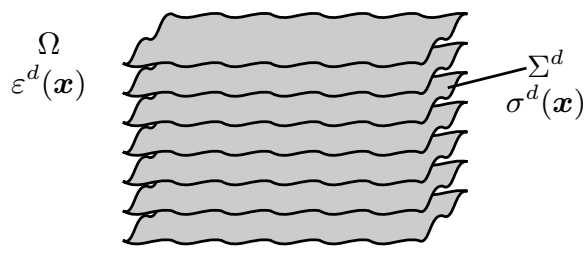

(b)

Figure 1: Geometry of the problem. (a) The unit cell, $Y=[0,1]^{3}$, with hypersurface $\Sigma_{0}$, (b) a layered structure consisting of parallel, conducting sheets $\Sigma^{d}$ equipped with a spatially dependent surface conductivity $\sigma^{d}(\boldsymbol{x})$. We assume that the layered structure is immersed in a (unbounded) medium with a spatially dependent permittivity $\varepsilon^{d}(\boldsymbol{x})$.

The actual proof assumes a more restrictive "reference configuration" which is described in Section 2 The geometry is shown in Figure 1. The (complex) surface conductivity $\sigma^{d}$ of every sheet, which is in principle frequency $(\omega-)$ dependent, has real and imaginary parts that can be tuned to allow for the propagation of surface plasmon-polaritons on the isolated sheet [20, 22].

The scaling parameter $d, d \ll 1$, describes the fine scale of the problem and in particular the distance separating the conducting sheets: Let $Y=[0,1]^{3}$ denote the unit cell and let $\Sigma_{0}$ denote a smooth hypersurface in $Y$ (with smooth, periodic continuation); see Figure 1 1 . We then define a union of stacked, disconnected hypersurfaces by

$$
\Sigma^{d}=\bigcup_{\boldsymbol{z} \in \mathbb{Z}^{n}} d\left(\boldsymbol{z}+\Sigma_{0}\right)
$$

see Figure 1b. For all $\boldsymbol{x} \in \Sigma^{d}$, the surface conductivity $\sigma^{d}(\boldsymbol{x})$ is a tensor acting on the tangent space $T_{\boldsymbol{x}} \Sigma^{d}$. We assume that $\sigma^{d}(\boldsymbol{x})$ exhibits both fine-scale (periodic) and large-scale variations in space. We also assume that $\sigma^{d} \sim d$ so that the total conductivity remains finite when $d \ll 1$. (This scaling is also consistent with the fact that $\left|\sigma^{d}\right|$ must be small enough for the appearance of a fine-scale surface plasmon-polariton on an isolated sheet [20, 22]). We thus write

$$
\sigma^{d}(\boldsymbol{x})=d \sigma(\boldsymbol{x}, \boldsymbol{x} / d)
$$

where $\sigma(\boldsymbol{x}, \boldsymbol{y})$ is a tensor acting on the tangent space $T_{\boldsymbol{y}} \Sigma_{0}$ for all $(\boldsymbol{x}, \boldsymbol{y}) \in$ $\mathbb{R}^{3} \times \Sigma_{0}$ and is independent of $d$ and periodic with respect to $\boldsymbol{y}$. Similarly, the permittivity of the ambient medium $\varepsilon^{d}(\boldsymbol{x})$, defined for $\boldsymbol{x} \in \mathbb{R}^{3} \backslash \Sigma^{d}$, is assumed to be given by

$$
\varepsilon^{d}(\boldsymbol{x})=\varepsilon(\boldsymbol{x}, \boldsymbol{x} / d)
$$

for some tensor $\varepsilon(\boldsymbol{x}, \boldsymbol{y})$ independent of $d$. The quantities $\varepsilon(\boldsymbol{x}, \boldsymbol{y})$ and $\sigma(\boldsymbol{x}, \boldsymbol{y})$ will be henceforth referred to as the rescaled permittivity and surface conductivity, respectively. Additional conditions on the domain, geometry and material parameters are provided below. 
We now consider time-harmonic Maxwell's equations written in the form

$$
\left\{\begin{aligned}
\nabla \times \boldsymbol{E}^{d} & =i \omega \mu \boldsymbol{H}^{d}, \\
\nabla \times \boldsymbol{H}^{d} & =-i \omega \varepsilon^{d} \boldsymbol{E}^{d}+\boldsymbol{J}_{a} .
\end{aligned}\right.
$$

Here, $\boldsymbol{E}^{d}$ and $\boldsymbol{H}^{d}$ denote the electric and magnetic field, respectively; and $\boldsymbol{J}_{a}$ is the (externally applied) source current density. The parameter $\mu$ denotes the magnetic permeability of the ambient space; e.g., $\mu=\mu_{0}$, a scalar constant, for the vacuum.

In order to write the boundary conditions across $\Sigma^{d}$, we introduce the normal unit vector field $\boldsymbol{\nu}(\boldsymbol{x})$ on $\Sigma^{d}$, and let $[.]_{\Sigma^{d}}$ denote the jump over $\Sigma^{d}$ :

$$
[\boldsymbol{F}]_{\Sigma^{d}}(\boldsymbol{x}):=\lim _{\alpha \searrow 0}(\boldsymbol{F}(\boldsymbol{x}+\alpha \boldsymbol{\nu})-\boldsymbol{F}(\boldsymbol{x}-\alpha \boldsymbol{\nu})) \quad \boldsymbol{x} \in \Sigma^{d} .
$$

We also denote by $\boldsymbol{F}_{T}$ the tangential component of any vector field $\boldsymbol{F}$, viz., $\boldsymbol{F}_{T}=(\boldsymbol{\nu} \times \boldsymbol{F}) \times \boldsymbol{\nu}$. The current density induced on the sheets because of the effect of $\sigma^{d}$ is $\boldsymbol{J}_{\Sigma^{d}}=\boldsymbol{\delta}_{\Sigma^{d}} \sigma^{d} \boldsymbol{E}_{T}^{d}$. Hence, the boundary conditions for the tangential components of the electromagnetic field across $\Sigma^{d}$ read [20, 22]

$$
\left\{\begin{array}{l}
{\left[\boldsymbol{\nu} \times \boldsymbol{E}^{d}\right]_{\Sigma^{d}}=0} \\
{\left[\boldsymbol{\nu} \times \boldsymbol{H}^{d}\right]_{\Sigma^{d}}=\sigma^{d} \boldsymbol{E}_{T}^{d} .}
\end{array}\right.
$$

\subsection{Main result}

We will show that as $d \rightarrow 0$, the electric and magnetic fields, $\boldsymbol{E}^{d}$ and $\boldsymbol{H}^{d}$, converge to the solutions, $\mathcal{E}$ and $\mathcal{H}$, of the homogenized system

$$
\left\{\begin{aligned}
\nabla \times \mathcal{E} & =i \omega \mu \mathcal{H} \\
\nabla \times \mathcal{H} & =-i \omega \varepsilon^{\mathrm{eff}} \mathcal{E}+\boldsymbol{J}_{a}
\end{aligned}\right.
$$

where the effective permittivity $\varepsilon^{\mathrm{eff}}=\varepsilon^{\mathrm{eff}}(\boldsymbol{x})$ is given as an appropriate average involving the $d$-independent (rescaled) permittivity and conductivity $\varepsilon$ and $\sigma$ :

$$
\begin{aligned}
\varepsilon^{\mathrm{eff}}(\boldsymbol{x}):=\int_{Y} \varepsilon(\boldsymbol{x}, \boldsymbol{y})\left(I_{3}+\nabla_{y} \chi(\boldsymbol{x}, \boldsymbol{y})\right) \mathrm{d} y & \\
& -\frac{1}{i \omega} \int_{\Sigma_{0}}\left(\sigma(\boldsymbol{x}, \boldsymbol{y}) P_{T}\left(I_{3}+\nabla_{y} \chi(\boldsymbol{x}, \boldsymbol{y})\right)\right) \mathrm{d} o_{y} .
\end{aligned}
$$

In the above, $I_{3}$ denotes the identity matrix in $\mathbb{R}^{3}, P_{T}$ is the projection matrix onto the tangent set of $\Sigma_{0}$ and $\nabla_{y} \boldsymbol{\chi}(\boldsymbol{x}, \boldsymbol{y})$ denotes the matrix $\left[\partial_{y_{i}} \chi_{j}(\boldsymbol{x}, \boldsymbol{y})\right](i, j=$ $1,2,3)$. The corrector $y \mapsto \boldsymbol{\chi}(\boldsymbol{x}, \boldsymbol{y})$ solves the following cell problem (for all $\boldsymbol{x}$ 
in some bounded open set $\Omega \subset \mathbb{R}^{3}$ ):

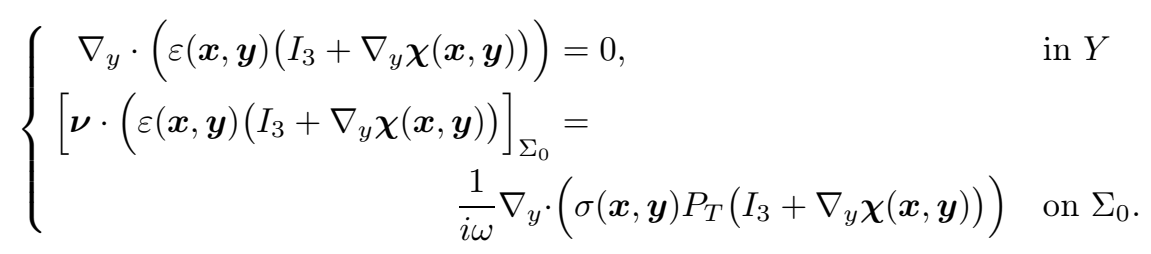

As mentioned in the introduction, this cell problem is similar to equation (34) in [4] for the case (scaling regime) of "strong interface layer". Note that even when $\varepsilon$ and $\sigma$ are scalars, the effective permittivity $\varepsilon^{\text {eff }}$ is a $3 \times 3$ matrix as typically expected for the case of a bulk material, that is, if $\sigma \equiv 0$ [17]. Under the assumptions of Theorem 2.4 (see (19)), we have

$$
\operatorname{Im}\left(\varepsilon^{\mathrm{eff}}(\boldsymbol{x}) \boldsymbol{\xi} \cdot \overline{\boldsymbol{\xi}}\right) \geq c\left(1+\frac{1}{\omega}\right)|\boldsymbol{\xi}|^{2},
$$

which ensures the well-posedness of homogenized system (3).

We alert the reader that the proofs in this paper are only developed in the scalar case, i.e., when the material parameters $\varepsilon^{d}$ and $\sigma^{d}$ are scalars, for the sake of simplicity. On the other hand, the above statements are written more generally, for tensorial $\varepsilon^{d}$ and $\sigma^{d}$. Our analysis can be extended to the tensor case without difficulties.

\subsection{Novelty and application}

The insertion of an array of metallic sheets, each of which can sustain surface plasmon-polaritons, into dielectric hosts with small enough interlayer spacing has significant physical appeal [21, 23, 24]. From an analysis perspective, this type of structure motivates the homogenization procedure of this paper, and leads to an intriguing homogenization result; cf. Section 1.2 .

Foremost, effective permittivity (4) is now the combination of two averages, namely, one average stemming from the ambient-medium permittivity tensor $\varepsilon(\boldsymbol{x}, \boldsymbol{y})$, and another from the surface conductivity $\sigma(\boldsymbol{x}, \boldsymbol{y})$ of each metallic sheet. To our knowledge, this combination of two effective parameters, one coming from a bulk property and another expressing the property of a hypersurface, has not occurred in most of the previous homogenization results; see, e.g., [1, 40]. An exception is the homogenization of Maxwell's equations in a two-phase composite material carried out in [4], which we mentioned above; see also the discussion in Sections 1.4, and 5.

For applications in plasmonics, the case with a surface conductivity, $\sigma^{d}$, that has a dominant imaginary part, viz., $\operatorname{Im} \sigma^{d} \gg \operatorname{Re} \sigma^{d}>0$ in the case of a scalar $\sigma^{d}$, has attracted particular attention. Such a surface conductivity can be created with novel 2D materials, for example graphene [13]. By carefully tuning the frequency, geometry, or the surface conductivity, $\sigma$, via doping of the $2 \mathrm{D}$

material, one may obtain $\varepsilon^{\text {eff }}$ with eigenvalues that have vanishing, or negative 
real part. The homogenized system described by (4) can thus be viewed as a metamaterial exhibiting highly unusual optical phenomena such as the epsilonnear-zero effect or negative refraction [21, 23, 24]. This implication and the connection of our homogenization result to existing predictions of epsilon-nearzero behavior are discussed in Section 5 .

\subsection{On past works}

Our analysis relies on firm concepts of homogenization theory $([1,6,611,29$, 32]), which we employ in the setting of electromagnetic wave propagation in the presence of $2 \mathrm{D}$ plasmonic materials. Over the past decade, numerous studies have been conducted on related applications, especially because of the prospect of fabricating metamaterials with unusual properties in nanophotonics. These properties come from combining and averaging out suitable microstructures. For recent reviews from an applied physics perspective, we refer the reader to [8, 16, 41].

From the viewpoint of analysis, we should highlight a number of homogenization results [3 5, 38 40] that are relevant to our problem formulation, as well as the germane notion of two-scale convergence [1, 29] which underlies our approach. In particular, the homogenization results obtained in [3, 5, 38[40] coincide with (44) in the special case with a vanishing surface conductivity, $\sigma(\boldsymbol{x}, \boldsymbol{y})=0$.

As mentioned above, our work is related to that in [4] which also analyzes the effect of surface currents in the homogenization of the time harmonic Maxwell equations. In [4], however, the authors focus on a different geometric and physical setting, which involves two-phase materials with certain inclusions. Various regimes, which depend on the strength of the interfacial currents, the wavelength and the skin depth, are studied in [4]. Both the mathematical formulation and the homogenized equations that we derive in the present paper are closely related to the case referred to as the strong interface layer in [4] (see Theorem 2 in [4]).

However, there are key differences in the geometric setting of the two problems. In [4] the authors consider a material (e.g., clay) containing periodically distributed rock inclusions; thus, the hypersurfaces are boundaries of small disconnected sets rather than the large 2D sheets studied here. Most notably, in [4], a key tool in [4] is the generalization of the notion of two-scale convergence to functions defined on periodic surfaces [2, 27], which exploits the geometric setting of small inclusions in a crucial way. The proof that we develop in the present paper, however, is directly motivated by our geometry consisting of large 2D interfaces and does not rely on this notion of two-scale convergence on surfaces but instead recovers directly the convergence of the currents to the appropriate term in the sense of distribution; cf. Proposition 4.4. We point out that in principle our results can be extended to the geometry of closed inclusions considerd in [4], and vice versa. In that sense we offer an independent proof of the homogenization result.

We should also mention a number of related results for periodic media that are obtained by use of the Bloch wave theory; see, e.g., [35] where the authors 
conclude that only a few Bloch waves effectively contribute to the macroscopic field. In a similar physical context, the application of homogenization to finite photonic crystals is described in [14]; and its connection to certain numerical multiscale methods for Maxwell's equations in composite materials is elaborated in [9, 15]. In particular, in [14] the authors formally apply a two-scale asymptotic expansion to derive effective bulk parameters that take into account the crystal boundary. To these works we add the homogenization of Maxwell's equations in the presence of rough boundaries and interfaces pioneered in [28].

\subsection{Outline}

The remainder of the paper is organized as follows. In Section 2, we introduce the weak formulation of the problem, along with two key theorems (Theorems 2.3 and 2.4) which permeate our analysis. Section 3 focuses on the proof of one of these theorems (Theorem 2.3), namely, the existence of a weak solution to Maxwell's equations for a finite microstructure scale, $d>0$. In Section 4, we prove the second key theorem (Theorem 2.4) which establishes the convergence of the weak solution for $d>0$ to the homogenization limit as $d \rightarrow 0$ and recovers the cell problem. Finally, in Section 5 we conclude our work with a discussion of its relevance to the design of plasmonic crystals with unusual optical properties. In Section 5, an outline of related open problems is given as well. Appendix A provides an overview of important results in two-scale convergence. Appendix B contains details of the extension of our analysis to more general hypersurfaces for the metallic sheets. At the risk of redundancy, we repeat that our analysis in this paper focuses on the scalar case. The extension of our proofs to tensorial parameters does not present any difficulties, and is not pursued here.

\section{Homogenization of layered structures}

In this section, we introduce a weak formulation of (1) and (2), and state the main theorems of this paper. At this stage, we will analyze a special choice of geometry ("reference configuration"), in which the hypersurfaces are flat. (We outline an extension of our proof to curved hypersurfaces in Appendix B. Specifically, let $\Omega$ be a bounded open set in $\mathbb{R}^{3}$ of the form $\Omega=\Sigma_{0} \times \Gamma$, where $\Sigma_{0}$ is a (bounded) subset of $\mathbb{R}^{2}$ and $\Gamma=(-L, L)$. The layered structure is then described by

$$
\Sigma^{d}=\bigcup_{k \in \Gamma^{d}} \Sigma_{0} \times\{k d\}
$$

where $\Gamma^{d}=\{k \in \mathbb{Z} ; k d \in(-L, L-d)\}$.

Remark 2.1. We point out that our homogenization result holds for a larger class of geometries. In fact, all theorems and proofs that are presented in this section can be readily extended to cases of periodic structures that are diffeomorphic to the above reference configuration (as depicted in Figure 1). We outline an extension of our proof to such curved hypersurfaces in Appendix B. 
We will use the notation $\boldsymbol{x}=\left(\boldsymbol{x}^{\prime}, x_{3}\right) \in \Sigma \times \Gamma$ when necessary. For later convenience, we also define $\tilde{\Gamma}^{d}=\bigcup_{k \in \Gamma^{d}}[k d,(k+1) d]$. Hence, we have

$$
(-L+d, L-d) \subset \tilde{\Gamma}^{d} \subset(-L, L) .
$$

\subsection{Weak formulation}

Formally, our problem is equivalent to the system

$$
\left\{\begin{aligned}
\nabla \times \boldsymbol{E}^{d} & =i \omega \mu \boldsymbol{H}^{d}, \\
\nabla \times \boldsymbol{H}^{d} & =-i \omega \varepsilon^{d} \boldsymbol{E}^{d}+\boldsymbol{J}^{d},
\end{aligned} \quad \text { in } \Omega\right.
$$

where

$$
\boldsymbol{J}^{d}=\left(\sigma^{d} \boldsymbol{E}_{T}^{d}\right) \delta_{\Sigma^{d}}+\boldsymbol{J}_{a} .
$$

Note that (7) implies the following Helmholtz-type equation for the electric field

$$
\frac{1}{\mu} \nabla \times\left(\nabla \times \boldsymbol{E}^{d}\right)=\omega^{2} \varepsilon^{d} \boldsymbol{E}^{d}+i \omega \boldsymbol{J}^{d},
$$

along with the relation $\boldsymbol{H}^{d}=\frac{1}{i \omega \mu} \nabla \times \boldsymbol{E}^{d}$. On the boundary of $\Omega$, we supplement this equation with the following impedance boundary condition [25]:

$$
\frac{1}{\mu}\left(\nabla \times \boldsymbol{E}^{d}\right) \times \nu=i \omega \lambda \boldsymbol{E}_{T}^{d} \quad \text { on } \partial \Omega .
$$

Recall that $\nu$ denotes the normal unit vector. Boundary condition (10) is often used for scattering configurations: For the particular choice $\lambda=\sqrt{\mu^{-1} \varepsilon}$, formula (10) recovers a first-order absorbing boundary condition [25].

By multiplying (9) by the conjugate of a smooth test function $\boldsymbol{\Psi}$, we obtain the following formulation for Maxwell's equations in domain $\Omega$ :

$$
\begin{aligned}
\int_{\Omega} \frac{1}{\mu}\left(\nabla \times \boldsymbol{E}^{d}\right) \cdot(\nabla \times \overline{\mathbf{\Psi}}) \mathrm{d} x+\int_{\partial \Omega} \frac{1}{\mu} \nu \times\left(\nabla \times \boldsymbol{E}^{d}\right) \cdot \overline{\mathbf{\Psi}}_{T} \mathrm{~d} x \\
=\int_{\Omega} \omega^{2} \varepsilon^{d} \boldsymbol{E}^{d} \cdot \overline{\mathbf{\Psi}} \mathrm{d} x+i \omega \int_{\Omega} \boldsymbol{J}^{d} \cdot \mathbf{\Psi} \mathrm{d} x .
\end{aligned}
$$

By substituting expression (8) for current $\boldsymbol{J}^{d}$ and using boundary condition (10) on $\partial \Omega$, we thus obtain the following weak formulation:

$$
\begin{aligned}
& \int_{\Omega} \frac{1}{\mu}(\left.\nabla \times \boldsymbol{E}^{d}\right) \cdot(\nabla \times \overline{\mathbf{\Psi}}) \mathrm{d} x-\int_{\partial \Omega} i \omega \lambda \boldsymbol{E}_{T}^{d} \cdot \overline{\mathbf{\Psi}}_{T} \mathrm{~d} o_{x} \\
& \quad=\int_{\Omega} \omega^{2} \varepsilon^{d} \boldsymbol{E}^{d} \cdot \overline{\mathbf{\Psi}} \mathrm{d} x+\int_{\Sigma^{d}} i \omega \sigma^{d} \boldsymbol{E}_{T}^{d} \cdot \overline{\mathbf{\Psi}}_{T} \mathrm{~d} o_{x}+\int_{\Omega} i \omega \boldsymbol{J}_{a} \cdot \overline{\mathbf{\Psi}} \mathrm{d} x .
\end{aligned}
$$

Remark 2.2. In order to obtain the desired a priori estimates below, we will need to assume that the bulk material (ambient medium) is dissipative. This property can be ensured by addition of the current density $\sigma_{0} \boldsymbol{E}^{d}$ in the bulk material, where $\sigma_{0}>0$. Alternatively, this current ensues by addition of the term $i \frac{\sigma_{0}}{\omega}$ to the permittivity $\varepsilon^{d}$. This is the reason why $\varepsilon^{d}$ will be complex valued, with strictly positive imaginary part, in this section (see also conditions (15) -(16) and Remark [2.5). 


\subsection{Function spaces}

The space $H(\operatorname{curl} ; \Omega)$ denotes the set of complex valued vector functions $\boldsymbol{u} \in L^{2}\left(\Omega ; \mathbb{C}^{3}\right)$ such that $\nabla \times \boldsymbol{u} \in L^{2}\left(\Omega ; \mathbb{C}^{3}\right)$. Given a hypersurface $\Lambda \subset \bar{\Omega}$, the trace $\nu \times\left.\boldsymbol{u}\right|_{\Lambda}$ (where $\nu$ is the unit normal vector to $\Lambda$ ) is well defined for functions in $H(\operatorname{curl} ; \Omega)$ and it belongs to $H^{-\frac{1}{2}}\left(\operatorname{div}_{\Lambda}, \Lambda\right)$. We denote by $\boldsymbol{u}_{T}=(\nu \times \boldsymbol{u}) \times \nu$ the tangential component of $\boldsymbol{u}$. In view of (11), the function space that is natural for our problem is

$$
X^{d}=\left\{\boldsymbol{u} \in H(\operatorname{curl} ; \Omega) ; \boldsymbol{u}_{T} \in L^{2}\left(\partial \Omega ; \mathbb{C}^{3}\right), \boldsymbol{u}_{T} \in L^{2}\left(\Sigma^{d} ; \mathbb{C}^{3}\right)\right\},
$$

equipped with the norm

$$
\|\boldsymbol{u}\|_{X^{d}}^{2}=\|\boldsymbol{u}\|_{L^{2}(\Omega)}^{2}+\|\nabla \times \boldsymbol{u}\|_{L^{2}(\Omega)}^{2}+\left\|\boldsymbol{u}_{T}\right\|_{L^{2}(\partial \Omega)}^{2}+\left\|\boldsymbol{u}_{T}\right\|_{L^{2}\left(\Sigma^{d}\right)}^{2},
$$

where

$$
\left\|\boldsymbol{u}_{T}\right\|_{L^{2}\left(\Sigma^{d}\right)}^{2}=d \int_{\Sigma^{d}}\left|\boldsymbol{u}_{T}\right|^{2} \mathrm{~d} o_{x}=\sum_{k \in \Gamma^{d}} d \int_{\Sigma}\left|\boldsymbol{u}_{T}\left(x^{\prime}, k d\right)\right|^{2} \mathrm{~d} x^{\prime}
$$

Note the presence of the factor $d$ in this last norm. This is a natural definition since with this scaling the $L^{2}\left(\Sigma^{d}\right)$ norm is a Riemann sum approximation of the $L^{2}(\Omega)$ norm when $d \ll 1$. With these prerequisites at hand we can introduce a precise problem formulation: Equation (11) is equivalent to

$$
\boldsymbol{E}^{d} \in X^{d}, \quad a^{d}\left(\boldsymbol{E}^{d}, \boldsymbol{\Psi}\right)=i \omega \int_{\Omega} \boldsymbol{J}_{a} \cdot \overline{\mathbf{\Psi}} \mathrm{d} x \quad \forall \boldsymbol{\Psi} \in X^{d},
$$

where the sesquilinear form $a^{d}: X^{d} \times X^{d} \rightarrow \mathbb{C}$ is defined by

$$
\begin{aligned}
a^{d}(\boldsymbol{u}, \boldsymbol{v})=\int_{\Omega} \frac{1}{\mu}(\nabla \times \boldsymbol{u}) \cdot(\nabla \times \overline{\boldsymbol{v}}) \mathrm{d} x-\int_{\Omega} \omega^{2} \varepsilon^{d} \boldsymbol{u} \cdot \overline{\boldsymbol{v}} \mathrm{d} x \\
\quad-\int_{\Sigma^{d}} i \omega \sigma^{d} \boldsymbol{u}_{T} \cdot \overline{\boldsymbol{v}}_{T} \mathrm{~d} o_{x}-\int_{\partial \Omega} i \omega \lambda \boldsymbol{u}_{T} \cdot \overline{\boldsymbol{v}}_{T} \mathrm{~d} o_{x} .
\end{aligned}
$$

We will show that (13) uniquely determines the electric field $\boldsymbol{E}^{d}$ (see Theorem 2.3). The corresponding magnetic field is then given by

$$
\boldsymbol{H}^{d}(\boldsymbol{x})=\frac{1}{i \omega \mu} \nabla \times \boldsymbol{E}^{d}(\boldsymbol{x}) .
$$

\subsection{Main theorems}

We are now ready to state our main results. Throughout the paper, we will make the following assumption on the properties of the material:

Assumption: We assume (for simplicity) that $\mu$ and $\lambda$ are positive, real, scalar constants and that $\varepsilon^{d}$ and $\sigma^{d}$ are measurable complex valued functions satisfying

$$
0<c \leq \operatorname{Im} \varepsilon^{d}(\boldsymbol{x}) \leq C \quad \text { and } \quad\left|\operatorname{Re} \varepsilon^{d}(\boldsymbol{x})\right| \leq C \quad \forall \boldsymbol{x} \in \Omega
$$


and

$$
0<c \leq \frac{1}{d} \operatorname{Re} \sigma^{d}(\boldsymbol{x}) \leq C \quad \text { and } \quad\left|\frac{1}{d} \operatorname{Im} \sigma^{d}(\boldsymbol{x})\right| \leq C \quad \forall \boldsymbol{x} \in \Sigma^{d},
$$

for some constants $c$ and $C$.

Our first result concerns the existence of a solution for the problem (13) when $d>0$ :

Theorem 2.3. Under the assumption above, system (13) has a unique solution in $X^{d}$ for all $\boldsymbol{J}_{a} \in L^{2}\left(\Omega ; \mathbb{C}^{3}\right)$ and for all $d>0$.

The proof of Theorem 2.3 is presented in Section 3 Next, we state our main convergence result for the homogenization limit that was formally discussed in Section 1.2 .

Theorem 2.4. Under the assumptions of Theorem [2.3, we also assume that

$$
\varepsilon^{d}(\boldsymbol{x})=\varepsilon(\boldsymbol{x}, \boldsymbol{x} / d), \quad \text { and } \quad \sigma^{d}(\boldsymbol{x})=d \sigma(\boldsymbol{x}, \boldsymbol{x} / d),
$$

where $\varepsilon(\boldsymbol{x}, \boldsymbol{y})$ and $\sigma(\boldsymbol{x}, \boldsymbol{y})$ are complex-valued scalar functions, periodic with respect to $\boldsymbol{y} \in Y$, and $\sigma$ is Lipschitz continuous:

$$
\left|\nabla_{x} \sigma(\boldsymbol{x}, \boldsymbol{y})\right|,\left|\nabla_{y} \sigma(\boldsymbol{x}, \boldsymbol{y})\right| \leq C \quad \forall(\boldsymbol{x}, \boldsymbol{y}) \in \Omega \times Y .
$$

Then, for all $\boldsymbol{J}_{a} \in L^{2}\left(\Omega ; \mathbb{C}^{3}\right)$, the electric field $\boldsymbol{E}^{d}(\boldsymbol{x})$ of (13) and the corresponding magnetic field $\boldsymbol{H}^{d}(\boldsymbol{x})$ defined by (14) converge weakly in $L^{2}\left(\Omega ; \mathbb{C}^{3}\right)$ to functions $\mathcal{E}(\boldsymbol{x})$ and $\mathcal{H}(\boldsymbol{x})$ satisfying the following weak form of the homogenized system (3) :

$$
\mathcal{E} \in X^{0}, \quad a^{0}(\mathcal{E}, \Psi)=\int_{\Omega} i \omega \boldsymbol{J}_{a} \cdot \bar{\Psi} \mathrm{d} x \quad \forall \boldsymbol{\Psi} \in X^{0},
$$

and

$$
\mathcal{H}(\boldsymbol{x})=\frac{1}{i \omega \mu} \nabla \times \mathcal{E}(\boldsymbol{x}) .
$$

Here, the sesquilinear form $a^{0}: X^{0} \times X^{0} \rightarrow \mathbb{C}$ is given by

$a^{0}(\boldsymbol{u}, \boldsymbol{v})=\int_{\Omega} \frac{1}{\mu}(\nabla \times \boldsymbol{u}) \cdot(\nabla \times \overline{\boldsymbol{v}}) \mathrm{d} x-\int_{\Omega} \omega^{2} \varepsilon^{e f f} \boldsymbol{u} \cdot \overline{\boldsymbol{v}} \mathrm{d} x-\int_{\partial \Omega} i \omega \lambda \boldsymbol{u}_{T} \cdot \overline{\boldsymbol{v}}_{T} \mathrm{~d} o_{x}$, and the space $X^{0}$ is defined by

$$
X^{0}=\left\{\boldsymbol{u} \in H(\operatorname{curl} ; \Omega) ; \boldsymbol{u}_{T} \in L^{2}\left(\partial \Omega ; \mathbb{C}^{3}\right)\right\} .
$$

The effective permittivity $\varepsilon^{\text {eff }}$ is given by (4) (with $P_{T}=\operatorname{diag}(1,1,0) \in \mathbb{R}^{3 \times 3}$ ).

Note that $\sigma(\boldsymbol{x}, \boldsymbol{y})$ is only really defined for $\boldsymbol{y} \in \Sigma_{0}$, but in our simple geometry we can treat it as a function defined for all $\boldsymbol{y} \in Y$ which is independent of $\boldsymbol{y}_{3}$. In particular the gradient $\nabla_{y} \sigma$ in (17) is a tangential gradient. In the framework of this theorem, conditions (15)-(16) are equivalent to

$$
c \leq \operatorname{Im} \varepsilon(\boldsymbol{x}, \boldsymbol{y}), \operatorname{Re} \sigma(\boldsymbol{x}, \boldsymbol{y}) \leq C, \quad|\varepsilon(\boldsymbol{x}, \boldsymbol{y})|,|\sigma(\boldsymbol{x}, \boldsymbol{y})| \leq C \quad \forall(\boldsymbol{x}, \boldsymbol{y}) \in \Omega \times Y .
$$

The proof of Theorem 2.4 is deferred to Section 4 
Remark 2.5. Theorems 2.3 and 2.4 also hold when $\mu, \varepsilon^{d}$ and $\sigma^{d}$ are matrix valued, provided all the respective assumptions are replaced with appropriate matrix inequalities. For example, the first conditions in (15)-(16) should be replaced by

$$
c|\xi|^{2} \leq \operatorname{Im}\left(\varepsilon^{d} \xi \cdot \bar{\xi}\right) \leq C|\xi|^{2}, \quad c|\xi|^{2} \leq \frac{1}{d} \operatorname{Re}\left(\sigma^{d} \xi \cdot \bar{\xi}\right) \leq C|\xi|^{2}, \quad \forall \xi \in \mathbb{R}^{3} .
$$

As a last step in this section, we establish the well-posedness of (18).

Theorem 2.6. Under the assumptions of Theorem 2.4, the homogenized problem (18) has a unique solution $\mathcal{E}(\boldsymbol{x})$ in $X^{0}$ for all $\boldsymbol{J}_{a} \in L^{2}\left(\Omega ; \mathbb{C}^{3}\right)$.

The proof of this theorem is deferred to the end of Section 3

\section{Proofs of Theorem 2.3 and Theorem 2.6}

In this section, we prove the existence of a solution for both the weak formulation of the problem for finite microstructure scale, $d>0$, as well as for homogenized problem (18). The proof is based on the Lax-Milgram theorem, which we recall here for the convenience of the reader:

Theorem 3.1 (Lax-Milgram Theorem). Let $X$ be a Hilbert space over $\mathbb{C}$ and let $b: X \times X \rightarrow \mathbb{C}$ be a sesquilinear form such that

$$
\begin{aligned}
& b \text { is bounded: } \quad|b(u, v)| \leq C_{1}\|u\|_{X}\|v\|_{X} \quad \forall u, v \in X, \\
& b \text { is coercive: } \quad \operatorname{Re} b(u, u) \geq c_{2}\|u\|_{X}^{2} \quad \forall u \in X \text {. }
\end{aligned}
$$

Then, for any $L: X \rightarrow \mathbb{C}$ linear and bounded, there exists a unique $u \in X$ such that

$$
b(u, \Psi)=L(\Psi) \quad \forall \Psi \in X .
$$

Proof of Theorem 2.3. In order to apply Theorem 3.1, we first replace the permeability $\mu \in \mathbb{R}$ by $\mu+i \eta$ for some small $\eta>0$ and consider the sesquilinear form $b_{\eta}^{d}(u, v)=i a^{d}(u, v)$. Indeed, we have

$$
\begin{aligned}
\operatorname{Re} b_{\eta}^{d}(\boldsymbol{u}, \boldsymbol{u})=\frac{\eta}{\mu^{2}+\eta^{2}} & \int_{\Omega}|\nabla \times \boldsymbol{u}|^{2} \mathrm{~d} x+\int_{\Omega} \omega^{2} \operatorname{Im}\left(\varepsilon^{d}\right)|\boldsymbol{u}|^{2} \mathrm{~d} x \\
& +d \int_{\Sigma^{d}} \omega \frac{1}{d} \operatorname{Re}\left(\sigma^{d}\right)\left|\boldsymbol{u}_{T}\right|^{2} \mathrm{~d} o_{x}+\int_{\partial \Omega} \omega \lambda\left|\boldsymbol{u}_{T}\right|^{2} \mathrm{~d} o_{x},
\end{aligned}
$$

and so assumptions (15)-(16) implies

$$
\operatorname{Re} b_{\eta}^{d}(\boldsymbol{u}, \boldsymbol{u}) \geq c_{\eta}\|\boldsymbol{u}\|_{X^{d}}^{2} \quad \forall \boldsymbol{u} \in X^{d},
$$

for some small constant $c_{\eta}>0$ depending on $\eta$ (and $d$ ). Furthermore, it is readily seen that assumptions (15)-(16) also gives

$$
\left|b_{\eta}^{d}(\boldsymbol{u}, \boldsymbol{v})\right| \leq C\|\boldsymbol{u}\|_{X^{d}}\|\boldsymbol{v}\|_{X^{d}}
$$


Hence, the Lax-Milgram theorem (Theorem 3.1) implies the existence of a unique $\boldsymbol{u}^{\eta}$ solution of

$$
\boldsymbol{u}^{\eta} \in X^{d}, \quad b_{\eta}^{d}\left(\boldsymbol{u}^{\eta}, \boldsymbol{v}\right)=-\int_{\Omega} \omega \boldsymbol{J}_{a} \cdot \overline{\boldsymbol{v}} \mathrm{d} x \quad \forall \boldsymbol{v} \in X^{d} .
$$

Next, we write

$$
b_{\eta}^{d}\left(\boldsymbol{u}^{\eta}, \boldsymbol{u}^{\eta}\right)=-\int_{\Omega} \omega \boldsymbol{J}_{a} \cdot \overline{\boldsymbol{u}}^{\eta} \mathrm{d} x,
$$

and by taking the real and imaginary parts of this relation, we obtain

$$
\begin{aligned}
& \int_{\Omega} \frac{\eta}{\mu^{2}+\eta^{2}}\left|\nabla \times \boldsymbol{u}^{\eta}\right|^{2} \mathrm{~d} x+\int_{\Omega} \omega^{2}\left(\operatorname{Im} \varepsilon^{d}\right)\left|\boldsymbol{u}^{\eta}\right|^{2} \mathrm{~d} x \\
& +d \int_{\Sigma^{d}} \omega\left(\frac{1}{d} \operatorname{Re} \sigma^{d}\right)\left|\boldsymbol{u}_{T}^{\eta}\right|^{2} \mathrm{~d} o_{y}+\int_{\partial \Omega} \omega \lambda\left|\boldsymbol{u}_{T}^{\eta}\right|^{2} \mathrm{~d} o_{x}=-\int_{\Omega} \omega \operatorname{Re}\left(\boldsymbol{J}_{a} \cdot \overline{\boldsymbol{u}}^{\eta}\right) \mathrm{d} x
\end{aligned}
$$

and

$$
\begin{aligned}
\int_{\Omega} \frac{\mu}{\mu^{2}+\eta^{2}} \mid \nabla \times & \left.\boldsymbol{u}^{\eta}\right|^{2} \mathrm{~d} x-\int_{\Omega} \omega^{2}\left(\operatorname{Re} \varepsilon^{d}\right)\left|\boldsymbol{u}^{\eta}\right|^{2} \mathrm{~d} x \\
& +d \int_{\Sigma^{d}} \omega\left(\frac{1}{d} \operatorname{Im} \sigma^{d}\right)\left|\boldsymbol{u}_{T}^{\eta}\right|^{2} \mathrm{~d} o_{x}=\int_{\Omega} \omega \operatorname{Im}\left(\boldsymbol{J}_{a} \cdot \overline{\boldsymbol{u}}^{\eta}\right) \mathrm{d} x .
\end{aligned}
$$

Using (15)-(16) and Young's inequality, we notice that (21) gives

$$
\left\|\boldsymbol{u}^{\eta}\right\|_{L^{2}(\Omega)}^{2}+\left\|\boldsymbol{u}_{T}^{\eta}\right\|_{L^{2}\left(\Sigma^{d}\right)}^{2}+\left\|\boldsymbol{u}_{T}^{\eta}\right\|_{L^{2}(\partial \Omega)}^{2} \leq C\left\|\boldsymbol{J}_{a}\right\|_{L^{2}(\Omega)}^{2},
$$

and the use of (15)-(16) and (22) combined with (23) in turn implies

$$
\left\|\nabla \times \boldsymbol{u}^{\eta}\right\|_{L^{2}(\Omega)}^{2} \leq C\left\|\boldsymbol{J}_{a}\right\|_{L^{2}(\Omega)}^{2}
$$

for some constant $C$ independent of $\eta$. Thus, we can pass to the limit $\eta \rightarrow 0$ in (20). This result completes the proof of Theorem 2.3.

The corresponding result for the homogenized system can be established in a similar vein.

Proof of Theorem [2.6. The well-posedness of (18) depends on the properties of the effective permittivity, $\varepsilon^{\text {eff }}$. We recall that formula (4) gives

$$
\begin{aligned}
\varepsilon_{i j}^{\mathrm{eff}}=\int_{Y} \varepsilon(\boldsymbol{x}, \boldsymbol{y})\left(\boldsymbol{e}_{j}+\nabla_{y} \boldsymbol{\chi}_{j}(\boldsymbol{x}, \boldsymbol{y})\right) \cdot \boldsymbol{e}_{i} \mathrm{~d} y \\
\quad-\frac{1}{i \omega} \int_{\Sigma_{0}}\left(\sigma(\boldsymbol{x}, \boldsymbol{y})\left(\boldsymbol{e}_{j}+\nabla_{y} \boldsymbol{\chi}_{j}(\boldsymbol{x}, \boldsymbol{y})\right)_{T} \cdot \boldsymbol{e}_{i T} \mathrm{~d} o_{y},\right.
\end{aligned}
$$


where $\chi(\boldsymbol{x}, \boldsymbol{y})$ solves the cell problem (5). Furthermore, by multiplying the cell problem by $\overline{\chi_{i}}$ and integrating over $Y$ (see also the weak formulation (45)), we obtain

$$
\begin{aligned}
\int_{Y} \varepsilon(\boldsymbol{x}, \boldsymbol{y})\left(\boldsymbol{e}_{j}\right. & \left.+\nabla_{y} \chi_{j}(\boldsymbol{x}, \boldsymbol{y})\right) \cdot \nabla_{y} \overline{\chi_{i}}(\boldsymbol{y}) \mathrm{d} y \\
& -\frac{1}{i \omega} \int_{\Sigma_{0}} \sigma(\boldsymbol{x}, \boldsymbol{y})\left(\boldsymbol{e}_{j}+\nabla_{y} \chi_{j}(\boldsymbol{x}, \boldsymbol{y})\right)_{T} \cdot\left(\nabla_{y} \overline{\chi_{i}}(\boldsymbol{y})\right)_{T} \mathrm{~d} o_{y}=0 .
\end{aligned}
$$

Combining the last two formulas, we arrive at the following alternative formula for $\varepsilon^{\text {eff: }}$

$$
\begin{aligned}
\varepsilon_{i j}^{\mathrm{eff}}= & \int_{Y} \varepsilon(\boldsymbol{x}, \boldsymbol{y})\left(\boldsymbol{e}_{j}+\nabla_{y} \boldsymbol{\chi}_{j}(\boldsymbol{x}, \boldsymbol{y})\right) \cdot\left(\boldsymbol{e}_{i}+\nabla_{y} \overline{\chi_{i}}(\boldsymbol{x}, \boldsymbol{y})\right) \mathrm{d} y \\
& -\frac{1}{i \omega} \int_{\Sigma_{0}}\left(\sigma(\boldsymbol{x}, \boldsymbol{y})\left(\boldsymbol{e}_{j}+\nabla_{y} \boldsymbol{\chi}_{j}(\boldsymbol{x}, \boldsymbol{y})\right)\right)_{T} \cdot\left(\boldsymbol{e}_{i}+\nabla_{y} \overline{\boldsymbol{\chi}_{i}}(\boldsymbol{x}, \boldsymbol{y})\right)_{T} \mathrm{~d} o_{y} .
\end{aligned}
$$

In this form, we see that when $\varepsilon$ and $\sigma$ are scalar (as in Theorem 2.4), we have

$$
\begin{aligned}
\varepsilon^{\mathrm{eff}} \boldsymbol{\xi} \cdot \overline{\boldsymbol{\xi}}=\int_{Y} \varepsilon(\boldsymbol{x}, \boldsymbol{y})\left|\boldsymbol{\xi}+\sum_{j=1}^{3} \nabla_{y} \chi_{j}(\boldsymbol{x}, \boldsymbol{y}) \xi_{j}\right|^{2} \mathrm{~d} y \\
\quad-\frac{1}{i \omega} \int_{\Sigma_{0}} \sigma(\boldsymbol{x}, \boldsymbol{y})\left|\left(\boldsymbol{\xi}+\sum_{j=1}^{3} \nabla_{y} \chi_{j}(\boldsymbol{x}, \boldsymbol{y}) \xi_{j}\right)_{T}\right|^{2} \mathrm{~d} y, \quad \forall \boldsymbol{\xi} \in \mathbb{R}^{3},
\end{aligned}
$$

and thus assumption (19) implies the inequality

$$
\operatorname{Im}\left(\varepsilon^{\mathrm{eff}}(\boldsymbol{x}) \boldsymbol{\xi} \cdot \overline{\boldsymbol{\xi}}\right) \geq c|\boldsymbol{\xi}|^{2} .
$$

We proceed exactly as in the proof of Theorem 2.3 (with inequality (25) playing the role of assumption (15)-(16)). This concludes the proof of Theorem 2.6.

\section{Prerequisites and proof of Theorem 2.4}

The core of this section is devoted to the two-scale convergence needed to establish our main homogenization result. In order to pass to the limit in (13) and prove Theorem 2.4 we must first establish an important a priori estimate as explained below (Section 4.1). The related machinery of two-scale convergence is utilized in Section 4.2. This leads to the proof of a main proposition for the homogenized system (Proposition 4.3 in Section 4.3); and the extraction of the requisite cell problem (Section 4.5). We conclude this section by proving Theorem 2.4, see Section 4.5.

\subsection{An a priori estimate}

We start with the following proposition, which establishes a crucial uniform a priori estimate that is used throughout the proof of this section. We recall that the norm $\|\cdot\|_{L^{2}\left(\Sigma^{d}\right)}$ is defined by (12) and includes a factor equal to $d$. 
Proposition 4.1. Under the assumptions of Theorem (2.4), the solution $\boldsymbol{E}^{d}$ of (13) is bounded in $X^{d}$ uniformly with respect to $d$. More precisely, there exists a constant $C$ independent of $d$ such that

$$
\frac{1}{\omega}\left\|\nabla \times \boldsymbol{E}^{d}\right\|_{L^{2}(\Omega)}^{2}+\omega\left\|\boldsymbol{E}^{d}\right\|_{L^{2}(\Omega)}^{2}+\left\|\boldsymbol{E}_{T}^{d}\right\|_{L^{2}\left(\Sigma^{d}\right)}^{2}+\left\|\boldsymbol{E}_{T}^{d}\right\|_{L^{2}(\partial \Omega)}^{2} \leq C\left\|\boldsymbol{J}_{a}\right\|_{L^{2}(\Omega)} .
$$

Proof. We take $\boldsymbol{\Psi}=\boldsymbol{E}^{d}$ in (13), to obtain

$$
\begin{aligned}
\int_{\Omega} \frac{1}{\mu}\left|\nabla \times \boldsymbol{E}^{d}\right|^{2} \mathrm{~d} x=\omega^{2} \int_{\Omega} \varepsilon & (\boldsymbol{x}, \boldsymbol{x} / d)\left|\boldsymbol{E}^{d}\right|^{2} \mathrm{~d} x+i \omega d \int_{\Sigma^{d}} \sigma(\boldsymbol{x}, \boldsymbol{x} / d)\left|\boldsymbol{E}_{T}^{d}\right|^{2} \mathrm{~d} o_{x} \\
& +i \omega \lambda \int_{\partial \Omega}\left|\boldsymbol{E}_{T}^{d}\right|^{2} \mathrm{~d} o_{x}+i \omega \int_{\Omega} \boldsymbol{J}_{a} \cdot \overline{\boldsymbol{E}^{d}} \mathrm{~d} x .
\end{aligned}
$$

Taking the real and imaginary part of (27) gives, respectively,

$$
\begin{aligned}
& \int_{\Omega} \frac{1}{\mu}\left|\nabla \times \boldsymbol{E}^{d}\right|^{2} \mathrm{~d} x=\omega^{2} \int_{\Omega} \operatorname{Re} \varepsilon(\boldsymbol{x}, \boldsymbol{x} / d)\left|\boldsymbol{E}^{d}\right|^{2} \mathrm{~d} x \\
&-\omega d \int_{\Sigma^{d}} \operatorname{Im} \sigma(\boldsymbol{x}, \boldsymbol{x} / d)\left|\boldsymbol{E}_{T}^{d}\right|^{2} \mathrm{~d} o_{x}-\omega \int_{\Omega} \operatorname{Im}\left(\boldsymbol{J}_{a} \cdot \overline{\boldsymbol{E}}^{d}\right) \mathrm{d} x,
\end{aligned}
$$

and

$$
\begin{array}{rl}
\omega^{2} \int_{\Omega} \operatorname{Im} \varepsilon(\boldsymbol{x}, \boldsymbol{x} / d)\left|\boldsymbol{E}^{d}\right|^{2} & \mathrm{~d} x+\omega d \int_{\Sigma^{d}} \operatorname{Re} \sigma(\boldsymbol{x}, \boldsymbol{x} / d)\left|\boldsymbol{E}_{T}^{d}\right|^{2} \mathrm{~d} o_{y} \\
& +\omega \lambda \int_{\partial \Omega}\left|\boldsymbol{E}_{T}^{d}\right|^{2} \mathrm{~d} o_{x}=-\omega \int_{\Omega} \operatorname{Re}\left(\boldsymbol{J}_{a} \cdot \overline{\boldsymbol{E}}^{d}\right) \mathrm{d} x .
\end{array}
$$

By invoking (19) and Young's inequality, we derive from (29) that

$$
\omega \int_{\Omega}\left|\boldsymbol{E}^{d}\right|^{2} \mathrm{~d} x+d \int_{\Sigma^{d}}\left|\boldsymbol{E}_{T}^{d}\right|^{2} \mathrm{~d} o_{y}+\lambda \int_{\partial \Omega}\left|\boldsymbol{E}_{T}^{d}\right|^{2} \mathrm{~d} o_{x} \leq C \int_{\Omega}\left|\boldsymbol{J}_{a}\right|^{2} \mathrm{~d} x .
$$

Furthermore, by virtue of (28) and (17) we infer that

$$
\int_{\Omega} \frac{1}{\mu}\left|\nabla \times \boldsymbol{E}^{d}\right|^{2} \mathrm{~d} x \leq C \omega \int_{\Omega}\left|\boldsymbol{J}_{a}\right|^{2} \mathrm{~d} x .
$$

The last two inequalities assert the desired result.

\subsection{Two-scale convergence}

The proof of our homogenization result (3) relies on the well-known notion of two-scale convergence (see [1, 29]). Several important results related to twoscale convergence are summarized in Appendix A for the convenience of the reader. Recall that the space $L_{\#}^{2}\left(Y ; \mathbb{C}^{k}\right)$ (respectively $\left.H_{\#}^{1}\left(Y ; \mathbb{C}^{k}\right)\right)$ denotes the closure under the $L^{2}$ norm (respectively $H^{1}$ norm) of the set $\mathbb{C}_{\#}^{\infty}\left(Y ; \mathbb{C}^{k}\right)$ of smooth $Y$-periodic functions defined on $\mathbb{R}^{3}$ with values in $\mathbb{C}^{k}$ (with $k=1$ or 3 ). 
Let $\boldsymbol{E}^{d}(\boldsymbol{x})$ be the unique solution of (13) as established by Theorem 2.3 We define $\boldsymbol{H}^{d}(\boldsymbol{x})$ by (14) for $\boldsymbol{x} \in \Omega$. Proposition 4.1 implies in particular that $\boldsymbol{H}^{d} \in L^{2}\left(\Omega ; \mathbb{C}^{3}\right)$. The weak formulation of (14) thus reads

$$
\int_{\Omega} \boldsymbol{E}^{d} \cdot(\nabla \times \overline{\mathbf{\Psi}}) \mathrm{d} x=i \omega \mu \int_{\Omega} \boldsymbol{H}^{d} \cdot \overline{\boldsymbol{\Psi}} \mathrm{d} x \quad \forall \boldsymbol{\Psi} \in H_{T}(\operatorname{curl} ; \Omega),
$$

where $H_{T}(\operatorname{curl} ; \Omega)=\left\{\boldsymbol{u} \in H(\operatorname{curl} ; \Omega) ; \boldsymbol{u}_{T}=0\right.$ on $\left.\partial \Omega\right\}$. Furthermore, in view of (14), we can now write (11) as

$$
\begin{aligned}
\int_{\Omega} \boldsymbol{H}^{d} \cdot( & \nabla \times \overline{\mathbf{\Psi}}) \mathrm{d} x-\int_{\partial \Omega} \lambda \boldsymbol{E}^{d} \cdot \overline{\boldsymbol{\Psi}}_{T} \mathrm{~d} o_{x}+\int_{\Omega} i \omega \varepsilon(\boldsymbol{x}, \boldsymbol{x} / d) \boldsymbol{E}^{d} \cdot \overline{\boldsymbol{\Psi}} \mathrm{d} x \\
= & d \int_{\Sigma^{d}} \sigma(\boldsymbol{x}, \boldsymbol{x} / d) \boldsymbol{E}_{T}^{d} \cdot \overline{\mathbf{\Psi}}_{T} \mathrm{~d} o_{x}+\int_{\Omega} \boldsymbol{J}_{a} \cdot \overline{\boldsymbol{\Psi}} \mathrm{d} x \quad \forall \boldsymbol{\Phi} \in X^{d} .
\end{aligned}
$$

Our goal is to pass to the limit $d \rightarrow 0$ in (30) and (31). We start with the following lemma.

Lemma 4.2. Up to a subsequence, the functions $\boldsymbol{E}^{d}(\boldsymbol{x})$ and $\boldsymbol{H}^{d}(\boldsymbol{x})$ two-scale converge to functions $\boldsymbol{E}^{(0)}(\boldsymbol{x}, \boldsymbol{y})$ and $\boldsymbol{H}^{(0)}(\boldsymbol{x}, \boldsymbol{y})$ in $L^{2}\left(\Omega ; L_{\#}^{2}\left(Y ; \mathbb{C}^{3}\right)\right)$ which satisfy

$$
\begin{aligned}
\boldsymbol{E}^{(0)}(\boldsymbol{x}, \boldsymbol{y}) & =\mathcal{E}(\boldsymbol{x})+\nabla_{y} \varphi(\boldsymbol{x}, \boldsymbol{y}), \\
\boldsymbol{H}^{(0)}(\boldsymbol{x}, \boldsymbol{y}) & =\mathcal{H}(\boldsymbol{x}),
\end{aligned}
$$

for some functions $\mathcal{E}, \mathcal{H} \in L^{2}\left(\Omega ; \mathbb{C}^{3}\right)$ and $\varphi(\boldsymbol{x}, \boldsymbol{y}) \in L^{2}\left(\Omega ; H_{\#}^{1}(Y, \mathbb{C})\right)$.

Proof. A proof of the Lemma can also be found in [38, 40]. Proposition 4.1 implies that the sequences $\boldsymbol{E}^{d}$ and $\boldsymbol{H}^{d}$ are both bounded in $L^{2}\left(\Omega ; \mathbb{C}^{3}\right)$. The classical two-scale convergence result (Theorem A.2) thus implies that there exist some subsequences, still denoted $\boldsymbol{E}^{d}$ and $\boldsymbol{H}^{d}$, which two-scale converge to $\boldsymbol{E}^{(0)}(\boldsymbol{x}, \boldsymbol{y})$ and $\boldsymbol{H}^{(0)}(\boldsymbol{x}, \boldsymbol{y})$, respectively.

We now consider a test function $\boldsymbol{\Psi}^{d}(\boldsymbol{x})=d w(\boldsymbol{x}) \boldsymbol{\Phi}(\boldsymbol{x} / d)$ with $\boldsymbol{\Phi} \in C_{\#}^{\infty}\left(Y ; \mathbb{C}^{3}\right)$ and $w \in \mathcal{D}(\Omega)$. Since $\boldsymbol{\Psi}^{d}=0$ on $\partial \Omega$, integration by parts entails

$$
\begin{aligned}
\int_{\Omega} \nabla \times \boldsymbol{E}^{d} \cdot \boldsymbol{\Psi}^{d} \mathrm{~d} x & =\int_{\Omega} \boldsymbol{E}^{d} \cdot \nabla \times \boldsymbol{\Psi}^{d} \mathrm{~d} x \\
& =\int_{\Omega} \boldsymbol{E}^{d}(\boldsymbol{x}) \cdot[d \nabla w(\boldsymbol{x}) \times \boldsymbol{\Phi}(\boldsymbol{x} / d)+w(\boldsymbol{x}) \nabla \times \boldsymbol{\Phi}(\boldsymbol{x} / d)] \mathrm{d} x .
\end{aligned}
$$

This equation can be re-arranged as

$$
\begin{aligned}
& \int_{\Omega} w(\boldsymbol{x}) \boldsymbol{E}^{d}(\boldsymbol{x}) \cdot \nabla \times \boldsymbol{\Phi}(\boldsymbol{x} / d) \mathrm{d} x \\
& \quad=d \int_{\Omega} \nabla \times \boldsymbol{E}^{d}(\boldsymbol{x}) \cdot \boldsymbol{\Phi}(\boldsymbol{x} / d) w(\boldsymbol{x}) \mathrm{d} x-d \int_{\Omega} \boldsymbol{E}^{d}(\boldsymbol{x}) \cdot \nabla w(\boldsymbol{x}) \times \mathbf{\Phi}(\boldsymbol{x} / d) \mathrm{d} x .
\end{aligned}
$$


Passing to the limit $(d \rightarrow 0)$ via stability estimate (26), we deduce that

$$
\int_{\Omega} w(\boldsymbol{x}) \int_{Y} \boldsymbol{E}^{(0)}(\boldsymbol{x}, \boldsymbol{y}) \cdot \nabla_{y} \times \boldsymbol{\Phi}(\boldsymbol{y}) \mathrm{d} y \mathrm{~d} x=0 .
$$

At this stage, introduce the homogenized electric field

$$
\mathcal{E}(\boldsymbol{x})=\int_{Y} \boldsymbol{E}^{(0)}(\boldsymbol{x}, \boldsymbol{y}) \mathrm{d} y .
$$

Equation (32) implies that the vector-valued function $\boldsymbol{f}(\boldsymbol{x}, \boldsymbol{y})=\boldsymbol{E}^{(0)}(\boldsymbol{x}, \boldsymbol{y})-$ $\left.\mathcal{E}(\boldsymbol{x}) \in L^{2}\left(\Omega ; L_{\#}^{2}\left(Y ; \mathbb{C}^{3}\right)\right)\right)$ satisfies

$$
\nabla_{y} \times \boldsymbol{f}(\boldsymbol{x}, \boldsymbol{y})=0 \quad \text { in } \mathcal{D}^{\prime}(\Omega \times Y), \quad \int_{Y} \boldsymbol{f}(\boldsymbol{x}, \boldsymbol{y}) \mathrm{d} y=0 \quad \text { for a. e. } \boldsymbol{x} \in \Omega .
$$

Utilizing a result from Fourier analysis (Lemma A.3), we conclude that there exists a scalar $\varphi(\boldsymbol{x}, \boldsymbol{y})$ in $L^{2}\left(\Omega ; H_{\#}^{1}(Y)\right)$ such that $f(\boldsymbol{x}, \boldsymbol{y})=\nabla_{y} \varphi(\boldsymbol{x}, \boldsymbol{y})$. Thus, we proved the first statement of Lemma 4.2. namely, that

$$
\boldsymbol{E}^{(0)}(\boldsymbol{x}, \boldsymbol{y})=\mathcal{E}(\boldsymbol{x})+\nabla_{y} \varphi(\boldsymbol{x}, \boldsymbol{y}) .
$$

In order to derive the corresponding result for $\boldsymbol{H}^{(0)}$, we first note that (31) and the bounds of Proposition 4.1 entail the estimate

$$
\left|\int_{\Omega} \boldsymbol{H}^{d} \cdot \nabla \times \boldsymbol{\Psi} \mathrm{d} x\right| \leq C\left\|\boldsymbol{J}_{a}\right\|_{L^{2}(\Omega)}\left\{\|\boldsymbol{\Psi}\|_{L^{2}(\Omega)}+\left\|\boldsymbol{\Psi}_{T}\right\|_{L^{2}(\partial \Omega)}+\left\|\boldsymbol{\Psi}_{T}\right\|_{L^{2}\left(\Sigma^{d}\right)}\right\} .
$$

By using a test function of the form $\boldsymbol{\Psi}^{d}(\boldsymbol{x})=d w(\boldsymbol{x}) \mathbf{\Phi}(\boldsymbol{x} / d)$ with $\boldsymbol{\Phi} \in C_{\#}^{\infty}\left(Y ; \mathbb{C}^{3}\right)$ and $w \in \mathcal{D}(\Omega)$, by analogy to (32) we obtain

$$
\int_{\Omega} w(\boldsymbol{x}) \int_{Y} \boldsymbol{H}^{(0)}(\boldsymbol{x}, \boldsymbol{y}) \cdot \nabla_{y} \times \boldsymbol{\Phi}(\boldsymbol{y}) \mathrm{d} x \mathrm{~d} y=0 .
$$

By proceeding as above, we now show that there exists a scalar function $\varphi_{1}(\boldsymbol{x}, \boldsymbol{y}) \in$ $L^{2}\left(\Omega ; H_{\#}^{1}(Y ; \mathbb{C})\right)$ such that

$$
\boldsymbol{H}^{(0)}(\boldsymbol{x}, \boldsymbol{y})=\mathcal{H}(\boldsymbol{x})+\nabla_{y} \varphi_{1}(\boldsymbol{x}, \boldsymbol{y}) .
$$

The substitution of test function $\Psi^{d}(\boldsymbol{x})=d \nabla(u(\boldsymbol{x}) v(\boldsymbol{x} / d))$ into (30) implies

$$
\begin{aligned}
0 & =d \int_{\Omega} \boldsymbol{H}^{d}(\boldsymbol{x}) \cdot \nabla(u(\boldsymbol{x}) v(\boldsymbol{x} / d)) \mathrm{d} x \\
& \left.=d \int_{\Omega} v(\boldsymbol{x} / d) \boldsymbol{H}^{d}(\boldsymbol{x}) \cdot \nabla_{x} u(\boldsymbol{x}) \mathrm{d} x+\int_{\Omega} u(\boldsymbol{x}) \boldsymbol{H}^{d}(\boldsymbol{x}) \cdot\left(\nabla_{y} v\right)(\boldsymbol{x} / d)\right) \mathrm{d} x .
\end{aligned}
$$

Passing to the limit $(d \rightarrow 0)$, we observe that

$$
\int_{\Omega} u(\boldsymbol{x}) \int_{Y} \boldsymbol{H}^{(0)}(\boldsymbol{x}, \boldsymbol{y}) \cdot \nabla_{y} v(\boldsymbol{y}) \mathrm{d} y \mathrm{~d} x=0 .
$$


This equality implies that $\nabla_{y} \cdot \boldsymbol{H}^{(0)}(\boldsymbol{x}, \boldsymbol{y})=0$ in the sense of distribution. Thus, by combining this result with (34) we conclude that

$$
\Delta_{y} \varphi_{1}(\boldsymbol{x}, \boldsymbol{y})=0 \text { in } \mathcal{D}^{\prime}(Y) \text { for a. e. } x \in \Omega .
$$

Together with the periodic boundary conditions $\left(\boldsymbol{y} \mapsto \varphi_{1}(\boldsymbol{x}, \boldsymbol{y})\right.$ is in $\left.H_{\#}^{1}(Y ; \mathbb{C})\right)$ this statement implies that $\varphi_{1}(\boldsymbol{x}, \boldsymbol{y})$ is constant and, thus, $\nabla_{y} \varphi_{1}=0$. Equality (34) now reduces to the equation $\boldsymbol{H}^{(0)}(\boldsymbol{x}, \boldsymbol{y})=\mathcal{H}(\boldsymbol{x})$. This result concludes the proof of Lemma 4.2.

\subsection{Derivation of the homogenized system}

We now fix a subsequence $\left\{d_{l}\right\}_{l \in \mathbb{N}}$ such that $\lim _{l \rightarrow \infty} d_{l}=0$ and the functions $\boldsymbol{E}^{d_{l}}(\boldsymbol{x})$ and $\boldsymbol{H}^{d_{l}}(\boldsymbol{x})$ two-scale converge to $\boldsymbol{E}^{(0)}(\boldsymbol{x}, \boldsymbol{y})=\mathcal{E}(\boldsymbol{x})+\nabla_{y} \varphi(\boldsymbol{x}, \boldsymbol{y})$ and $\boldsymbol{H}^{(0)}(\boldsymbol{x}, \boldsymbol{y})=\mathcal{H}(\boldsymbol{x})$ as in Lemma 4.2. We denote

$$
\boldsymbol{E}^{l}(\boldsymbol{x}):=\boldsymbol{E}^{d_{l}}(\boldsymbol{x}), \quad \boldsymbol{H}^{l}(\boldsymbol{x}):=\boldsymbol{H}^{d_{l}}(\boldsymbol{x}) .
$$

We will then prove that the limits $\mathcal{E}(x)$ and $\mathcal{H}(x)$ solve the homogenized problem (33). More precisely, the main result that we establish in this section is the following:

Proposition 4.3. The functions $\boldsymbol{E}^{l}(\boldsymbol{x})$ and $\boldsymbol{H}^{l}(\boldsymbol{x})$ converge weakly in $L^{2}\left(\Omega ; \mathbb{C}^{3}\right)$ to $\mathcal{E}(x)$ and $\mathcal{H}(x)$, where $\mathcal{E} \in X^{0}$ and $\mathcal{H} \in L^{2}\left(\Omega ; \mathbb{C}^{3}\right)$ satisfy

$$
\begin{gathered}
\int_{\Omega} \boldsymbol{H}(\boldsymbol{x}) \cdot(\nabla \times \overline{\mathbf{\Psi}}(\boldsymbol{x})) \mathrm{d} x-\int_{\partial \Omega} \lambda \mathcal{E}_{T}(\boldsymbol{x}) \cdot \overline{\boldsymbol{\Psi}}_{T}(\boldsymbol{x}) \mathrm{d} o_{x} \\
+\int_{\Omega} \int_{Y} i \omega \varepsilon(\boldsymbol{x}, \boldsymbol{y})\left(\mathcal{E}(\boldsymbol{x})+\nabla_{y} \varphi(\boldsymbol{x}, \boldsymbol{y})\right) \cdot \overline{\boldsymbol{\Psi}}(\boldsymbol{x}) \mathrm{d} y \mathrm{~d} x \\
-\int_{\Omega} \int_{\Sigma_{0}} \sigma(\boldsymbol{x}, \boldsymbol{y})\left(\mathcal{E}(\boldsymbol{x})+\nabla_{y} \varphi(\boldsymbol{x}, \boldsymbol{y})\right)_{T} \cdot \overline{\boldsymbol{\Psi}}_{T}(\boldsymbol{x}) \mathrm{d} o_{y} \mathrm{~d} x=\int_{\Omega} \boldsymbol{J}_{a}(\boldsymbol{x}) \cdot \overline{\mathbf{\Psi}}(\boldsymbol{x}) \mathrm{d} x,
\end{gathered}
$$

for all $\boldsymbol{\Psi} \in X^{0}$, and

$$
\int_{\Omega} \mathcal{E} \cdot(\nabla \times \overline{\mathbf{\Psi}}) \mathrm{d} x=i \omega \mu \int_{\Omega} \mathcal{H} \cdot \overline{\mathbf{\Psi}} \mathrm{d} x \quad \forall \boldsymbol{\Psi} \in H_{T}(\operatorname{curl} ; \Omega) .
$$

Furthermore, the corrector $\varphi(\boldsymbol{x}, \boldsymbol{y}) \in L^{2}\left(\Omega ; H_{\#}^{1}(Y, \mathbb{C})\right)$ satisfies $\nabla_{y} \varphi(\boldsymbol{x}, \boldsymbol{y})_{T} \in$ $L^{2}\left(\Omega \times \Sigma_{0}\right)$ and is determined by the following problem:

$$
\begin{aligned}
& i \omega \int_{Y} \varepsilon(\boldsymbol{x}, \boldsymbol{y})\left(\mathcal{E}(\boldsymbol{x})+\nabla_{y} \varphi(\boldsymbol{x}, \boldsymbol{y})\right) \cdot \nabla_{y} \bar{v}(\boldsymbol{y}) \mathrm{d} y \\
& \quad=\int_{\Sigma_{0}} \sigma(\boldsymbol{x}, \boldsymbol{y})\left(\mathcal{E}(\boldsymbol{x})+\nabla_{y} \varphi(\boldsymbol{x}, \boldsymbol{y})\right)_{T} \cdot \nabla_{T} \bar{v}(\boldsymbol{y}) \mathrm{d} o_{y} \quad \forall v \in H_{\#}^{1}(Y ; \mathbb{C})
\end{aligned}
$$

and for a. e. $x \in \Omega$. In the above, $\Sigma_{0}$ denotes the hypersurface $\left\{y_{3}=0\right\}$ in $Y$. 
In order to prove Proposition 4.3 we have to pass to the limit in (30) and (31), as $d_{l} \rightarrow 0$. While this procedure is relatively straightforward for (30), the passage to the limit in (31) is more delicate because of the presence of surface integrals on $\Sigma^{d}$. We will prove the following key proposition that establishes the limit of the requisite surface integrals:

Proposition 4.4. The corrector $\varphi$ satisfies $\nabla_{y} \varphi(\boldsymbol{x}, \boldsymbol{y})_{T} \in L^{2}\left(\Omega \times \Sigma_{0}\right)$, and for all functions $\boldsymbol{F}(\boldsymbol{x}, \boldsymbol{y})$ defined in $\Omega \times Y$ that are periodic with respect to $y$ and admit $\boldsymbol{F}, \nabla_{x} \boldsymbol{F}, \nabla_{y} \boldsymbol{F} \in L^{\infty}(\Omega \times Y)$ there holds:

$$
\begin{aligned}
\lim _{k \rightarrow \infty} d_{l} \int_{\Sigma^{d_{l}}} \boldsymbol{E}_{T}^{l}(\boldsymbol{x}) \cdot \boldsymbol{F}_{T}\left(\boldsymbol{x}, \boldsymbol{x} / d_{l}\right) \mathrm{d} o_{x}= \\
\int_{\Omega} \int_{\Sigma_{0}}\left(\mathcal{E}(\boldsymbol{x})+\nabla_{y} \varphi(\boldsymbol{x}, \boldsymbol{y})\right)_{T} \cdot F_{T}(\boldsymbol{x}, \boldsymbol{y}) \mathrm{d} o_{y} \mathrm{~d} x .
\end{aligned}
$$

The proof of this proposition is deferred to Section 4.4

Proof of Proposition 4.3. The choice of the subsequence $\boldsymbol{E}^{l}(\boldsymbol{x})$ and $\boldsymbol{H}^{l}(\boldsymbol{x})$ together with classical two-scale convergence results (Theorem A.2) imply that $\boldsymbol{E}^{l}(\boldsymbol{x})$ and $\boldsymbol{H}^{l}(\boldsymbol{x})$ converge weakly (in $L^{2}\left(\Omega ; \mathbb{C}^{3}\right)$ ) to the functions $\mathcal{E}(\boldsymbol{x})$ and $\mathcal{H}(\boldsymbol{x})$. Furthermore, Proposition 4.1 implies that $\mathcal{E}(\boldsymbol{x})$ is in $X^{0}$.

Passing to the limit in (30), as $d_{l} \rightarrow 0$, we obtain

$$
\int_{\Omega} \mathcal{E} \cdot(\nabla \times \overline{\mathbf{\Psi}}) \mathrm{d} x=i \omega \mu \int_{\Omega} \mathcal{H} \cdot \overline{\mathbf{\Psi}} \mathrm{d} x \quad \forall \boldsymbol{\Psi} \in H_{T}(\operatorname{curl} ; \Omega),
$$

which implies (36).

Next, the estimate of Proposition 4.1 implies that $\nabla \times \boldsymbol{E}^{l}$ is bounded in $L^{2}(\Omega)$ and thus converges weakly in $L^{2}(\Omega)$ to $\nabla \times \mathcal{E}$. Furthermore, Proposition 4.1 also implies that $\left.\boldsymbol{E}_{T}^{l}\right|_{\partial \Omega}$ is bounded in $L^{2}(\partial \Omega)$. In particular, there is a subsequence $\left.\boldsymbol{E}_{T}^{l^{\prime}}\right|_{\partial \Omega}$ which converges weakly in $L^{2}(\partial \Omega)$ and for any smooth test function $\Psi$ we have:

$$
\begin{aligned}
\int_{\partial \Omega} \boldsymbol{E}_{T}^{l^{\prime}} \cdot(\overline{\boldsymbol{\Psi}} \times \nu) \mathrm{d} o_{x} & =\int_{\Omega}\left(\nabla \times \boldsymbol{E}^{l^{\prime}}\right) \cdot \overline{\boldsymbol{\Psi}}-\boldsymbol{E}^{l^{\prime}} \cdot(\nabla \times \overline{\mathbf{\Psi}}) d x \\
& \rightarrow \int_{\Omega}(\nabla \times \mathcal{E}) \cdot \overline{\mathbf{\Psi}}-\mathcal{E} \cdot(\nabla \times \overline{\mathbf{\Psi}}) d x \\
& =\int_{\partial \Omega} \mathcal{E}_{T} \cdot(\overline{\mathbf{\Psi}} \times \nu) \mathrm{d} o_{x} .
\end{aligned}
$$

We deduce that this weak limit is $\left.\mathcal{E}_{T}\right|_{\partial \Omega}$. Since the limit is independent of the subsequence $l^{\prime}$, the whole sequence $\left.\boldsymbol{E}_{T}^{l}\right|_{\partial \Omega}$ converges weakly, viz.,

$$
\left.\left.\boldsymbol{E}_{T}^{l}\right|_{\partial \Omega} \rightarrow \mathcal{E}_{T}\right|_{\partial \Omega} \quad \text { weakly in } L^{2}(\partial \Omega) .
$$

We can now pass to the limit in (31). By invoking the usual properties of two-scale convergence in combination with the results of Proposition 4.4 and 
the choice $\boldsymbol{F}(\boldsymbol{x}, \boldsymbol{y})=\sigma(\boldsymbol{x}, \boldsymbol{y}) \overline{\mathbf{\Psi}}(\boldsymbol{x}, \boldsymbol{y})$, where $\boldsymbol{\Psi}(\boldsymbol{x}, \boldsymbol{y})$ is a smooth vector-valued test function, we deduce that

$$
\begin{gathered}
\int_{\Omega} \boldsymbol{H}(\boldsymbol{x}) \cdot(\nabla \times \overline{\mathbf{\Psi}}(\boldsymbol{x})) \mathrm{d} x-\int_{\partial \Omega} \lambda \mathcal{E}_{T}(\boldsymbol{x}) \cdot \overline{\boldsymbol{\Psi}}_{T}(\boldsymbol{x}) \mathrm{d} o_{x} \\
+\int_{\Omega} \int_{Y} i \omega \varepsilon(\boldsymbol{x}, \boldsymbol{y})\left(\mathcal{E}(\boldsymbol{x})+\nabla_{y} \varphi(\boldsymbol{x}, \boldsymbol{y})\right) \cdot \overline{\boldsymbol{\Psi}}(\boldsymbol{x}) \mathrm{d} y \mathrm{~d} x \\
=\int_{\Omega} \int_{\Sigma_{0}} \sigma(\boldsymbol{x}, \boldsymbol{y})\left(\mathcal{E}(\boldsymbol{x})+\nabla_{y} \varphi(\boldsymbol{x}, \boldsymbol{y})\right)_{T} \cdot \overline{\boldsymbol{\Psi}}_{T}(\boldsymbol{x}) \mathrm{d} o_{y} \mathrm{~d} x+\int_{\Omega} \boldsymbol{J}_{a}(\boldsymbol{x}) \cdot \overline{\boldsymbol{\Psi}}(\boldsymbol{x}) \mathrm{d} x,
\end{gathered}
$$

which in turn gives (35).

Finally, by taking $\boldsymbol{\Psi}(\boldsymbol{x})=d_{l} \nabla\left(u(\boldsymbol{x}) v\left(\boldsymbol{x} / d_{l}\right)\right)$ as a smooth test function in (31), as well as by using Proposition 4.4 with $\boldsymbol{F}(\boldsymbol{x}, \boldsymbol{y})=u(\boldsymbol{x}) \nabla_{y} v(\boldsymbol{y})$ we pass to the limit (as $\left.d_{l} \rightarrow 0\right)$ :

$$
\begin{aligned}
\int_{\Omega} \bar{u}(x) \int_{Y} i \omega \varepsilon(\boldsymbol{x}, \boldsymbol{y})\left(\mathcal{E}(\boldsymbol{x})+\nabla_{y} \varphi(\boldsymbol{x}, \boldsymbol{y})\right) \cdot \nabla_{y} \bar{v}(\boldsymbol{y}) \mathrm{d} y \mathrm{~d} x \\
\quad=\int_{\Omega} \bar{u}(x) \int_{\Sigma_{0}} \sigma(\boldsymbol{x}, \boldsymbol{y})\left(\mathcal{E}(\boldsymbol{x})+\nabla_{y} \varphi(\boldsymbol{x}, \boldsymbol{y})\right)_{T} \cdot \nabla_{T} \bar{v}(\boldsymbol{y}) \mathrm{d} o_{y} \mathrm{~d} x
\end{aligned}
$$

which is the weak formulation of cell problem (37). This concludes the proof of Proposition 4.3 ,

\subsection{Proof of Proposition 4.4}

To simplify the notations in this proof, we drop the $l$ dependence of the subsequence, although it is understood that all limits are taken along the subsequence $d_{l}$.

Recalling our notation $\boldsymbol{x}=\left(\boldsymbol{x}^{\prime}, x_{3}\right) \in \Sigma \times \Gamma$, we note that

$$
\begin{aligned}
d \int_{\Sigma^{d}} \boldsymbol{E}_{T}^{d}(\boldsymbol{x}) \cdot \boldsymbol{F}_{T}(\boldsymbol{x}, \boldsymbol{x} / d) \mathrm{d} o_{x} & =\sum_{l \in \Gamma^{d}} d \int_{\Sigma} \boldsymbol{E}_{T}^{d}\left(\boldsymbol{x}^{\prime}, k d\right) \cdot \boldsymbol{F}_{T}\left(\boldsymbol{x}^{\prime}, k d ; \boldsymbol{x}^{\prime} / d, k\right) \mathrm{d} x^{\prime} \\
& =\sum_{k \in \Gamma^{d}} d \int_{\Sigma} \boldsymbol{E}_{T}^{d}\left(\boldsymbol{x}^{\prime}, k d\right) \cdot \boldsymbol{F}_{T}\left(\boldsymbol{x}^{\prime}, k d ; \boldsymbol{x}^{\prime} / d, 0\right) \mathrm{d} x^{\prime} .
\end{aligned}
$$

Here, the second equality stems from the fact that $\boldsymbol{F}_{T}$ is $y_{3}$-periodic with period 1. We now introduce the function

$$
\alpha^{d}(t)=\sum_{k \in \Gamma^{d}} d \int_{\Sigma} \boldsymbol{E}_{T}^{d}\left(\boldsymbol{x}^{\prime},(k+t) d\right) \cdot \boldsymbol{F}_{T}\left(\boldsymbol{x}^{\prime},(k+t) d ; \boldsymbol{x}^{\prime} / d, 0\right) \mathrm{d} x^{\prime},
$$

defined for $t \in[0,1)$. We point out that we only have $\boldsymbol{E}_{T}^{d} \in H^{-1 / 2}(\Sigma)$, so the integral above is not well defined in the classical sense. However, $F$ is a smooth test function, so we can make sense of the integral as a duality bracket $\langle\cdot, \cdot\rangle_{H^{-1 / 2}, H^{1 / 2}}$. The limit that we need to characterize in order to prove Proposition 4.4 is $\lim _{d \rightarrow 0} \alpha^{d}(0)$. The desired result will ensue from the following two lemmas. 
Lemma 4.5. Under the assumptions of Proposition 4.4, the function $\alpha^{d}$ satisfies

$$
\begin{aligned}
\left\|\alpha^{d}\right\|_{L^{2}(0,1)} & \leq C\|\boldsymbol{F}\|_{L^{\infty}(\Omega)}\left\|\boldsymbol{E}^{d}\right\|_{L^{2}(\Omega)} \\
& \leq C\|\boldsymbol{F}\|_{L^{\infty}(\Omega)}\left\|\boldsymbol{J}_{a}\right\|_{L^{2}(\Omega)}
\end{aligned}
$$

and is thus bounded in $L^{2}(0,1)$. Furthermore, when $d \rightarrow 0$, it converges weakly in $L^{2}(0,1)$ to the function

$$
\alpha^{0}(t):=\int_{\Omega} \int_{Y^{\prime}} \boldsymbol{E}_{T}^{(0)}\left(\boldsymbol{x} ; \boldsymbol{y}^{\prime}, t\right) \cdot \boldsymbol{F}_{T}\left(\boldsymbol{x} ; \boldsymbol{y}^{\prime}, 0\right) \mathrm{d} y^{\prime} \mathrm{d} x,
$$

where $Y^{\prime}=[0,1]^{2}$.

Lemma 4.6. Under the assumptions of Proposition 4.4, there exists a constant $C$ (depending on $F$, but independent of $d$ ) such that

$$
\begin{aligned}
\left\|\frac{d \alpha^{d}}{d t}\right\|_{L^{2}(0,1)}^{2} \leq & C\left(1+d^{2}\right)\left\|\boldsymbol{E}^{d}\right\|_{L^{2}(\Omega)}^{2} \\
& +C d^{2}\left(\left\|\nabla \times \boldsymbol{E}^{d}\right\|_{L^{2}(\Omega)}^{2}+\left\|\boldsymbol{E}^{d}\right\|_{L^{2}(\partial \Omega)}^{2}\right) \\
\leq & C\left(1+d^{2}\right)\left\|\boldsymbol{J}_{a}\right\|_{L^{2}(\Omega)}^{2}
\end{aligned}
$$

Proof of Proposition 4.4. Deferring the proofs of the last two lemmas to the end of the present section, we note that Lemma 4.6 implies that $\alpha^{d}(t)$ is bounded in $C^{1 / 2}(0,1)$ by virtue of the Sobolev embedding theorem and thus the convergence established in Lemma 4.5 is uniform. In particular, $\alpha^{0}(t)$ is defined pointwise with

$$
\alpha^{0}(0)=\int_{\Omega} \int_{\Sigma_{0}}\left(E^{(0)}(\boldsymbol{x}, \boldsymbol{y})\right)_{T} \cdot(F(\boldsymbol{x}, \boldsymbol{y}))_{T} \mathrm{~d} o_{y} \mathrm{~d} x
$$

(recall that $\left.\Sigma_{0}=Y^{\prime} \times\{0\}\right)$ and

$$
\lim _{d \rightarrow 0} \alpha^{d}(0)=\int_{\Omega} \int_{\Sigma_{0}}\left(E^{(0)}(\boldsymbol{x}, \boldsymbol{y})\right)_{T} \cdot(F(\boldsymbol{x}, \boldsymbol{y}))_{T} \mathrm{~d} o_{y} \mathrm{~d} x
$$

which is (38). Note also that

$$
\alpha^{d}(0)=d \int_{\Sigma^{d}} \boldsymbol{E}_{T}^{d}(\boldsymbol{x}) \cdot \boldsymbol{F}_{T}(\boldsymbol{x}, \boldsymbol{x} / d) \mathrm{d} o_{x} \leq C\left(d \int_{\Sigma^{d}}\left|\boldsymbol{F}_{T}(\boldsymbol{x}, \boldsymbol{x} / d)\right|^{2} \mathrm{~d} o_{x}\right)^{1 / 2} .
$$

Passing to the limit of this expression (using Lemma 2.4 in [2] and noting that under the assumptions of Proposition 4.4 we have $\boldsymbol{F}_{T} \in W^{1, \infty}(\Omega \times Y) \subset C(\Omega \times$ $Y)$ ), we deduce that

$$
\alpha^{0}(0) \leq C\left(\int_{\Omega} \int_{\Sigma_{0}}\left|\boldsymbol{F}_{T}(\boldsymbol{x}, \boldsymbol{y})\right|^{2} \mathrm{~d} o_{y} d x\right)^{1 / 2} .
$$

It follows that $\left(E^{(0)}(\boldsymbol{x}, \boldsymbol{y})\right)_{T} \in L^{2}\left(\Omega \times \Sigma_{0}\right)$ and thus $\nabla_{y} \varphi(\boldsymbol{x}, \boldsymbol{y})_{T} \in L^{2}\left(\Omega \times \Sigma_{0}\right)$, which completes the proof of Proposition 4.4. 
We return to the task of proving Lemmas 4.5 and 4.6 .

Proof of Lemma 4.5. Note that to prove (411), we can first assume that $\boldsymbol{E}^{d}$ is smooth enough for the all the integrals below to make sense and conclude that (41) holds by a density argument. We have:

$$
\begin{aligned}
\int_{0}^{1}\left|\alpha^{d}(t)\right|^{2} d t & \leq C\|\boldsymbol{F}\|_{L^{\infty}}^{2} \int_{0}^{1}\left|\sum_{k \in \Gamma^{d}} d \int_{\Sigma}\right| \boldsymbol{E}_{T}^{d}\left(\boldsymbol{x}^{\prime},(k+t) d\right)\left|\mathrm{d} x^{\prime}\right|^{2} \mathrm{~d} t \\
& \leq C\|\boldsymbol{F}\|_{L^{\infty}}^{2}\left|\tilde{\Gamma}^{d}\right| \int_{0}^{1} \sum_{k \in \Gamma^{d}} d\left|\int_{\Sigma}\right| \boldsymbol{E}_{T}^{d}\left(\boldsymbol{x}^{\prime},(k+t) d\right)\left|\mathrm{d} x^{\prime}\right|^{2} \mathrm{~d} t \\
& \leq C\|\boldsymbol{F}\|_{L^{\infty}}^{2}|\Gamma||\Sigma| \sum_{k \in \Gamma^{d}} d \int_{0}^{1} \int_{\Sigma}\left|\boldsymbol{E}_{T}^{d}\left(\boldsymbol{x}^{\prime},(k+t) d\right)\right|^{2} \mathrm{~d} x^{\prime} \mathrm{d} t \\
& \leq C\|\boldsymbol{F}\|_{L^{\infty}}^{2}|\Gamma||\Sigma| \sum_{k \in \Gamma^{d}} \int_{k d}^{(k+1) d} \int_{\Sigma}\left|\boldsymbol{E}_{T}^{d}\left(\boldsymbol{x}^{\prime}, x_{3}\right)\right|^{2} \mathrm{~d} x^{\prime} \mathrm{d} x_{3} \\
& \leq C\|\boldsymbol{F}\|_{L^{\infty}}^{2}|\Gamma||\Sigma| \int_{\tilde{\Gamma}^{d}} \int_{\Sigma}\left|\boldsymbol{E}_{T}^{d}\left(\boldsymbol{x}^{\prime}, x_{3}\right)\right|^{2} \mathrm{~d} x^{\prime} \mathrm{d} x_{3} .
\end{aligned}
$$

Recall that $\tilde{\Gamma}^{d}=\bigcup_{k \in \Gamma^{d}}[k d,(k+1) d]$ satisfies $(-L+d, L-d) \subset \tilde{\Gamma}^{d} \subset \Gamma=$ $(-L, L)$. This implies (41) and using Proposition 4.1] we get

$$
\left\|\alpha^{d}\right\|_{L^{2}(0,1)} \leq C\|\boldsymbol{F}\|_{L^{\infty}}\left\|\boldsymbol{E}^{d}\right\|_{L^{2}(\Omega)} \leq C\|\boldsymbol{F}\|_{L^{\infty}}\left\|\boldsymbol{J}_{a}\right\|_{L^{2}(\Omega)} .
$$

The last inequality entails in particular that, up to another subsequence, $\alpha^{d}$ converges weakly in $L^{2}(0,1)$ to a function $\alpha^{0}$. Furthermore, for any 1-periodic test function $\varphi: \mathbb{R} \rightarrow \mathbb{C}$, we assert that

$$
\begin{aligned}
\int_{0}^{1} \alpha^{d}(t) \varphi(t) \mathrm{d} t & =\sum_{k \in \Gamma^{d}} d \int_{0}^{1} \int_{\Sigma} \boldsymbol{E}_{T}^{d}\left(\boldsymbol{x}^{\prime},(k+t) d\right) \cdot \boldsymbol{F}_{T}\left(\boldsymbol{x}^{\prime},(k+t) d ; \boldsymbol{x}^{\prime} / d, 0\right) \varphi(t) \mathrm{d} x^{\prime} \mathrm{d} t \\
& =\sum_{k \in \Gamma^{d}} \int_{k d}^{(k+1) d} \int_{\Sigma} \boldsymbol{E}_{T}^{d}\left(\boldsymbol{x}^{\prime}, x_{3}\right) \cdot \boldsymbol{F}_{T}\left(\boldsymbol{x}^{\prime}, x_{3} ; \boldsymbol{x}^{\prime} / d, 0\right) \varphi\left(x_{3} / d\right) \mathrm{d} x^{\prime} \mathrm{d} x_{3} \\
& =\int_{\tilde{\Gamma}^{d}} \int_{\Sigma} \boldsymbol{E}_{T}^{d}\left(\boldsymbol{x}^{\prime}, x_{3}\right) \cdot \boldsymbol{F}_{T}\left(\boldsymbol{x}^{\prime}, x_{3} ; \boldsymbol{x}^{\prime} / d, 0\right) \varphi\left(x_{3} / d\right) \mathrm{d} x^{\prime} \mathrm{d} x_{3} \\
& =\int_{\Sigma \times \tilde{\Gamma}^{d}} \boldsymbol{E}_{T}^{d}(\boldsymbol{x}) \cdot \boldsymbol{F}_{T}\left(\boldsymbol{x} ; \boldsymbol{x}^{\prime} / d, 0\right) \varphi\left(x_{3} / d\right) \mathrm{d} x .
\end{aligned}
$$

Using the definition of two-scale convergence and (6), we see that

$$
\begin{aligned}
\lim _{d \rightarrow 0} \int_{0}^{1} \alpha^{d}(t) \varphi(t) \mathrm{d} t & =\int_{\Omega} \int_{Y} \boldsymbol{E}_{T}^{(0)}(\boldsymbol{x}, \boldsymbol{y}) \cdot \boldsymbol{F}_{T}\left(\boldsymbol{x} ; \boldsymbol{y}^{\prime}, 0\right) \varphi\left(y_{3}\right) \mathrm{d} y \mathrm{~d} x \\
& =\int_{0}^{1}\left(\int_{\Omega} \int_{Y^{\prime}} \boldsymbol{E}_{T}^{(0)}(\boldsymbol{x}, \boldsymbol{y}) \cdot \boldsymbol{F}_{T}\left(\boldsymbol{x} ; \boldsymbol{y}^{\prime}, 0\right) \mathrm{d} y^{\prime} \mathrm{d} x\right) \varphi\left(y_{3}\right) \mathrm{d} y_{3} .
\end{aligned}
$$


The uniqueness of the limit implies that the whole original subsequence converges to $\alpha^{0}$, which completes the proof.

We conclude this section with the proof of Lemma 4.6. We note that this proof is the only instance in which the special geometry of our framework (the fact that $\Omega=\Sigma \times(-L, L)$ where $\Sigma$ is a flat hypersurface) plays a significant role. A generalization of this result to geometries with non-flat hypersurfaces is given in the Appendix B.

Proof of Lemma 4.6. We prove (42) by first assuming that $\boldsymbol{E}^{d}$ is smooth enough for the all the integrals below to make sense (and conclude that (42) holds for our $\boldsymbol{E}^{d}$ by a density argument). We start with the formula

$$
\begin{aligned}
\frac{\mathrm{d} \alpha^{d}}{\mathrm{~d} t}(t)= & \sum_{k \in \Gamma^{d}} d^{2} \int_{\Sigma} \partial_{x_{3}} \boldsymbol{E}_{T}^{d}\left(\boldsymbol{x}^{\prime},(k+t) d\right) \cdot \boldsymbol{F}_{T}\left(\boldsymbol{x}^{\prime},(k+t) d ; \boldsymbol{x}^{\prime} / d, 0\right) \mathrm{d} x^{\prime} \\
& +\sum_{k \in \Gamma^{d}} d^{2} \int_{\Sigma} \boldsymbol{E}_{T}^{d}\left(\boldsymbol{x}^{\prime},(k+t) d\right) \cdot\left[\partial_{x_{3}} \boldsymbol{F}_{T}\right]\left(\boldsymbol{x}^{\prime},(k+t) d ; \boldsymbol{x}^{\prime} / d, 0\right) \mathrm{d} x^{\prime} \\
= & : \beta_{1}(t)+\beta_{2}(t) .
\end{aligned}
$$

Now consider the second term, $\beta_{2}(t)$. Using the fact that $\partial_{x_{3}} \boldsymbol{F} \in L^{\infty}$, we have

$$
\begin{aligned}
\int_{0}^{1}\left|\beta_{2}(t)\right|^{2} d t & \leq\left\|\partial_{x_{3}} \boldsymbol{F}\right\|_{L^{\infty}}^{2} d^{2} \int_{0}^{1}\left(\sum_{k \in \Gamma^{d}} d \int_{\Sigma}\left|\boldsymbol{E}_{T}^{d}\left(\boldsymbol{x}^{\prime},(k+t) d\right)\right| \mathrm{d} x^{\prime}\right)^{2} \mathrm{~d} t \\
& \leq\left\|\partial_{x_{3}} \boldsymbol{F}\right\|_{L^{\infty}}^{2}|\Gamma| d^{2} \int_{0}^{1} \sum_{k \in \Gamma^{d}} d\left(\int_{\Sigma}\left|\boldsymbol{E}_{T}^{d}\left(\boldsymbol{x}^{\prime},(k+t) d\right)\right| \mathrm{d} x^{\prime}\right)^{2} \mathrm{~d} t \\
& \leq\left\|\partial_{x_{3}} \boldsymbol{F}\right\|_{L^{\infty}}^{2}|\Gamma| d^{2}|\Sigma| \int_{0}^{1} \sum_{k \in \Gamma^{d}} d \int_{\Sigma}\left|\boldsymbol{E}_{T}^{d}\left(\boldsymbol{x}^{\prime},(k+t) d\right)\right|^{2} \mathrm{~d} x^{\prime} \mathrm{d} t \\
& \leq\left\|\partial_{x_{3}} \boldsymbol{F}\right\|_{L^{\infty}}^{2}|\Gamma| d^{2}|\Sigma| \sum_{k \in \Gamma^{d}} d \int_{\Sigma} \int_{0}^{1}\left|\boldsymbol{E}_{T}^{d}\left(\boldsymbol{x}^{\prime},(k+t) d\right)\right|^{2} \mathrm{~d} t \mathrm{~d} x^{\prime} \\
& \leq\left\|\partial_{x_{3}} \boldsymbol{F}\right\|_{L^{\infty}}^{2}|\Gamma| d^{2}|\Sigma| \sum_{k \in \Gamma^{d}} \int_{k d}^{(k+1) d} \int_{\Sigma}\left|\boldsymbol{E}_{T}^{d}\left(\boldsymbol{x}^{\prime}, x_{3}\right)\right|^{2} \mathrm{~d} x_{3} \mathrm{~d} x^{\prime} \\
& \leq\left\|\partial_{x_{3}} \boldsymbol{F}\right\|_{L^{\infty}}^{2}|\Gamma| d^{2}|\Sigma| \int_{\Gamma^{\prime}} \int_{\Sigma}\left|\boldsymbol{E}_{T}^{d}\left(\boldsymbol{x}^{\prime}, x_{3}\right)\right|^{2} \mathrm{~d} x^{\prime} \mathrm{d} x_{3} \\
& \leq|\Omega| d^{2}\left\|\partial_{x_{3}} \boldsymbol{F}\right\|_{L^{\infty}}^{2}\left\|\boldsymbol{E}^{d}\right\|_{L^{2}(\Omega)}^{2} \cdot
\end{aligned}
$$

To determine a bound for $\beta_{1}(t)$, we use the fact that the derivative $\partial_{x_{3}} \boldsymbol{E}_{T}^{d}$ is a combination of $\nabla \times \boldsymbol{E}^{d}$ and $\nabla_{T} \boldsymbol{E}_{3}^{d}$. After expanding the dot product in $\beta_{1}(t)$, we end up with two similar terms (involving $\partial_{x_{3}} \boldsymbol{E}_{1}^{d} \boldsymbol{F}_{1}$ and $\partial_{x_{3}} \boldsymbol{E}_{2}^{d} \boldsymbol{F}_{2}$ respectively), and we will find a bound for the first one only (the second term is handled in 
the same way):

$$
\begin{aligned}
\beta_{11}(t) & :=\sum_{k \in \Gamma^{d}} d^{2} \int_{\Sigma} \partial_{x_{3}} \boldsymbol{E}_{1}^{d}\left(\boldsymbol{x}^{\prime},(k+t) d\right) \boldsymbol{F}_{1}\left(\boldsymbol{x}^{\prime},(k+t) d, \boldsymbol{x}^{\prime} / d, 0\right) \mathrm{d} x^{\prime} \\
& =\sum_{k \in \Gamma^{d}} d^{2} \int_{\Sigma} \partial_{x_{3}} \boldsymbol{E}_{1}^{d}\left(\boldsymbol{x}^{\prime},(k+t) d\right) w_{k}^{d}\left(\boldsymbol{x}^{\prime}, t\right) \mathrm{d} x^{\prime}
\end{aligned}
$$

where $w_{k}^{d}\left(\boldsymbol{x}^{\prime}, t\right)=\boldsymbol{F}_{1}\left(\boldsymbol{x}^{\prime},(k+t) d, \boldsymbol{x}^{\prime} / d, 0\right)$. Using the definition of the curl and integration by parts once, we can then write

$$
\begin{aligned}
\beta_{11}(t)=\sum_{k \in \Gamma^{d}} d^{2} \int_{\Sigma}\left(\nabla \times \boldsymbol{E}^{d}\right)_{2}\left(\boldsymbol{x}^{\prime},(k+t) d\right) w_{k}^{d}\left(\boldsymbol{x}^{\prime}, t\right) \mathrm{d} x^{\prime} \\
\quad+\sum_{k \in \Gamma^{d}} d^{2} \int_{\Sigma} \partial_{x_{1}} \boldsymbol{E}_{3}^{d}\left(\boldsymbol{x}^{\prime},(k+t) d\right) w_{k}^{d}\left(\boldsymbol{x}^{\prime}, t\right) \mathrm{d} x^{\prime} \\
=\sum_{k \in \Gamma^{d}} d^{2} \int_{\Sigma}\left(\nabla \times \boldsymbol{E}^{d}\right)_{2}\left(\boldsymbol{x}^{\prime},(k+t) d\right) w_{k}^{d}\left(\boldsymbol{x}^{\prime}, t\right) \mathrm{d} x^{\prime} \\
-\sum_{k \in \Gamma^{d}} d^{2} \int_{\Sigma} \boldsymbol{E}_{3}^{d}\left(\boldsymbol{x}^{\prime},(k+t) d\right) \partial_{x_{1}}\left[w_{k}^{d}\left(\boldsymbol{x}^{\prime}, t\right)\right]_{\Sigma} \mathrm{d} x^{\prime} \\
\quad+\sum_{k \in \Gamma^{d}} d^{2} \int_{\partial \Sigma} \boldsymbol{E}_{3}^{d}\left(\boldsymbol{x}^{\prime},(k+t) d\right) w_{k}^{d}\left(\boldsymbol{x}^{\prime}, t\right) \boldsymbol{\nu}_{1} \mathrm{~d} o_{x}^{\prime} .
\end{aligned}
$$

By using the estimates

$$
\left\|w_{k}^{d}\left(\boldsymbol{x}^{\prime}, t\right)\right\|_{L^{\infty}} \leq\left\|\boldsymbol{F}_{1}\right\|_{L^{\infty}}, \quad \text { and } \quad\left\|\partial_{x_{1}} w_{k}^{d}\left(\boldsymbol{x}^{\prime}, t\right)\right\|_{L^{\infty}} \leq\left\|\nabla_{x} \boldsymbol{F}_{1}\right\|_{L^{\infty}}+\frac{1}{d}\left\|\nabla_{y} \boldsymbol{F}_{1}\right\|_{L^{\infty}},
$$

we conclude that (proceeding similarly to the case with the bound for $\beta_{2}(t)$ above)

$$
\begin{gathered}
\int_{0}^{1}\left|\beta_{11}(t)\right|^{2} \mathrm{~d} t \leq C d^{2}|\Gamma||\Sigma| \int_{\Gamma} \int_{\Sigma}\left|\left(\nabla \times \boldsymbol{E}^{d}\right)_{2}\left(\boldsymbol{x}^{\prime}, x_{3}\right)\right|^{2} \mathrm{~d} x^{\prime} \mathrm{d} x_{3} \\
+\left(C d^{2}+C\right)|\Gamma||\Sigma| \int_{\Gamma} \int_{\Sigma}\left|\boldsymbol{E}_{3}^{d}\left(\boldsymbol{x}^{\prime}, x_{3}\right)\right|^{2} \mathrm{~d} x^{\prime} \mathrm{d} x_{3} \\
+C d^{2}|\Gamma||\partial \Sigma| \int_{\Gamma} \int_{\partial \Sigma}\left|\boldsymbol{E}_{3}^{d}\left(\boldsymbol{x}^{\prime}, x_{3}\right) \nu_{1}\right|^{2} \mathrm{~d} o_{x}^{\prime} \mathrm{d} x_{3} .
\end{gathered}
$$

We notice that $\boldsymbol{E}_{3}^{d}$ is part of $\boldsymbol{E}_{T}^{d}$ on the boundary $\partial \Sigma \times \Gamma \subset \partial \Omega$; thus, we have

$$
\begin{gathered}
\int_{0}^{1}\left|\beta_{11}(t)\right|^{2} \mathrm{~d} t \leq C|\Omega| d^{2}\left\|\nabla \times \boldsymbol{E}^{d}\right\|_{L^{2}(\Omega)}^{2}+C|\partial \Omega| d^{2}\left\|\boldsymbol{E}^{d}\right\|_{L^{2}(\partial \Omega)}^{2} \\
+C|\Omega|\left(1+d^{2}\right)\left\|\boldsymbol{E}^{d}\right\|_{L^{2}(\Omega)}^{2}
\end{gathered}
$$

By combining these estimates, we obtain (42). The last inequality in Lemma4.6 then follows from Proposition 4.1 . 
4.5. The cell problem and proof of Theorem 2.4

We now turn our attention to the cell problem (37). We will prove the following proposition:

Proposition 4.7. Given $\mathcal{E}(x) \in L^{2}\left(\Omega ; \mathbb{C}^{3}\right)$, the cell problem (40) has a unique solution $\varphi(\boldsymbol{x}, \boldsymbol{y})$ satisfying $\varphi \in L^{2}\left(\Omega ; H_{\#}^{1}\left(Y ; \mathbb{C}^{3}\right)\right)$, and $\left(\nabla_{y} \varphi\right)_{T} \in L^{2}\left(\Omega \times \Sigma_{0}\right)$. Furthermore, we can write

$$
\varphi(\boldsymbol{x}, \boldsymbol{y})=\sum_{j=1}^{3} \chi_{j}(\boldsymbol{x}, \boldsymbol{y}) \mathcal{E}_{j}(\boldsymbol{x}),
$$

where for a. e. $\boldsymbol{x} \in \Omega, \boldsymbol{y} \mapsto \boldsymbol{\chi}_{j}(\boldsymbol{x}, \boldsymbol{y})$ is the unique solution in

$$
H=\left\{u \in H_{\#}^{1}\left(Y ; \mathbb{C}^{3}\right) ;\left(\nabla_{y} u\right)_{T} \in L^{2}\left(\Sigma_{0}\right)\right\}
$$

of

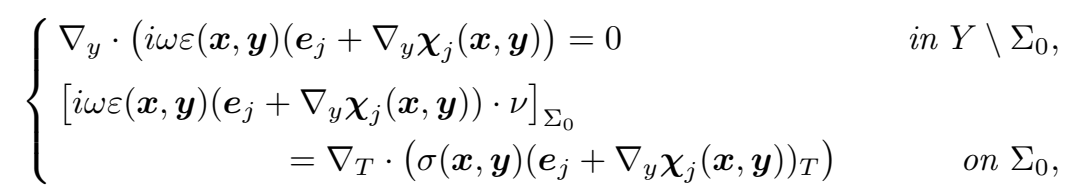

and satisfies $\chi_{j} \in L^{\infty}(\Omega ; H)$.

Proof. We first prove the existence and uniqueness of $\chi_{j}$ for $j=1,2,3$. This implies the existence of $\varphi(\boldsymbol{x}, \boldsymbol{y})$ given by (43). The uniqueness of $\varphi$ can be proved with exactly the same procedure as that for $\chi_{j}$.

The weak formulation of (44) reads

$$
\begin{aligned}
\int_{Y} i \omega \varepsilon(\boldsymbol{x}, \boldsymbol{y})\left(\boldsymbol{e}_{j}+\nabla_{y} \chi_{j}(\boldsymbol{x}, \boldsymbol{y})\right) \cdot \nabla_{y} \bar{v}(\boldsymbol{y}) \mathrm{d} y \\
=\int_{\Sigma_{0}} \sigma(\boldsymbol{x}, \boldsymbol{y})\left(\boldsymbol{e}_{j}+\nabla_{y} \chi_{j}(\boldsymbol{x}, \boldsymbol{y})\right)_{T} \cdot \nabla_{T} \bar{v}(\boldsymbol{y}) \mathrm{d} o_{y} .
\end{aligned}
$$

We note that $\boldsymbol{x}$ plays the role of a parameter here. Thus, for a fixed $\boldsymbol{x} \in \Omega$, we find the function $\boldsymbol{y} \mapsto \chi_{j}(\boldsymbol{x}, \boldsymbol{y})$ by solving

$$
b_{\boldsymbol{x}}\left(\chi_{j}(x, \cdot), v\right)=\int_{Y} i \omega \varepsilon(\boldsymbol{x}, \boldsymbol{y}) \boldsymbol{e}_{j} \cdot \nabla_{y} \bar{v}(\boldsymbol{y}) \mathrm{d} y-\int_{\Sigma_{0}} \sigma(\boldsymbol{x}, \boldsymbol{y})\left(\boldsymbol{e}_{j}\right)_{T} \cdot \nabla_{T} \bar{v}(\boldsymbol{y}) \mathrm{d} o_{y},
$$

with the sesquilinear form $b_{x}$ defined by

$b_{\boldsymbol{x}}(u, v):=\int_{Y}(-i \omega \varepsilon(\boldsymbol{x}, \boldsymbol{y})) \nabla_{y} u(\boldsymbol{y}) \cdot \nabla_{y} \bar{v}(\boldsymbol{y}) \mathrm{d} y+\int_{\Sigma_{0}} \sigma(\boldsymbol{x}, \boldsymbol{y}) \nabla_{T} u(\boldsymbol{y}) \cdot \nabla_{T} \bar{v}(\boldsymbol{y}) \mathrm{d} o_{y}$,

for all functions $u(\boldsymbol{y})$ and $v(\boldsymbol{y})$ in $H$. Under the assumptions of Theorem 2.4 the form $b_{x}$ is continuous and coercive on $H$ equipped with the norm

$$
\|u\|_{H}^{2}=\int_{Y}\left|\nabla_{y} u(\boldsymbol{y})\right|^{2} \mathrm{~d} y+\int_{\Sigma_{0}}\left|\nabla_{T} u(\boldsymbol{y})\right|^{2} \mathrm{~d} o_{y} .
$$


In particular, the coercivity follows from assumption (19):

$$
\begin{array}{r}
\operatorname{Re}\left(b_{\boldsymbol{x}}(u, u)\right)=\int_{Y} \operatorname{Im} \varepsilon(\boldsymbol{x}, \boldsymbol{y})\left|\nabla_{y} u(\boldsymbol{y})\right|^{2} \mathrm{~d} y+\int_{\Sigma_{0}} \operatorname{Re} \sigma(\boldsymbol{x}, \boldsymbol{y})\left|\nabla_{T} u(\boldsymbol{y})\right|^{2} \mathrm{~d} o_{y} \\
\geq c\|u\|_{H}^{2} .
\end{array}
$$

The existence and uniqueness of $\chi_{j}$ thus follows by virtue of the Lax-Milgram theorem 3.1. The bound in $L^{\infty}(\Omega ; H)$ follows from (47).

We are finally in a position to prove our main homogenization result:

Proof of Theorem 2.4. Let $d_{l}$ be any subsequence such that $\boldsymbol{E}^{d_{l}}$ and $\boldsymbol{H}^{d_{l}}$ twoscale converge as in Lemma 4.2. Then, using Proposition 4.3 we see that $\boldsymbol{E}^{d_{l}}$ and $\boldsymbol{H}^{d_{l}}$ converge weakly in $L^{2}\left(\Omega ; \mathbb{C}^{3}\right)$ to $\mathcal{E}(x) \in X^{0}$ and $\mathcal{H}(x) \in L^{2}\left(\Omega ; \mathbb{C}^{3}\right)$. Furthermore, by inserting the representation (43), which holds true by virtue of Proposition 4.7, into the weak formulation (35) of Proposition 4.3, we conclude that $\mathcal{E}$ solves the homogenized equation (18). The uniqueness of this limit, provided by Theorem 2.6, then implies that the whole sequences $\boldsymbol{E}^{d}(\boldsymbol{x})$ and $\boldsymbol{H}^{d}(\boldsymbol{x})$ are in fact convergent thus proving Theorem 2.4. Note that the fact that the corrector $\boldsymbol{\chi}(\boldsymbol{x}, \boldsymbol{y})$ solves (5) is an immediate consequence of Proposition 4.7

\section{Conclusion and discussion}

In this paper, we rigorously derived an effective description for electromagnetic wave propagation in a plasmonic crystal consisting of metallic sheets immersed in a non-magnetic dielectric medium. The main result of our analysis is a formula for the macroscopic dielectric permittivity, $\varepsilon^{\text {eff }}$, that combines a bulk average pertaining to the microstructure of the ambient medium and a surface average that takes into account the surface conductivity of each sheet. The accompanying corrector field is subject to a cell problem in which the divergence of the (microscale) dielectric permittivity enters as forcing along with a jump condition across the sheet that is proportional to the surface conductivity and involves the surface Laplacian of the corrector. In our analysis, we made use of the well-known notion of two-scale convergence from [1, 29].

It is worthwhile to compare our approach and main result to the ones in [4]. Although that work ([4]) reports a similar result for the effective permittivity, the geometric setting (in the context of geophysics) in [4] is different from ours. The mathematical formulations bear a resemblance; the respective proofs, however, are quite different. In [4], a key tool is the generalization of the notion of two-scale convergence to functions defined on periodic surfaces [2, 27]. This immediately implies the two-scale convergence of the interfacial currents $\left(\sigma^{d} \boldsymbol{E}_{T}^{d}\right) \delta_{\Sigma^{d}}$, and the difficulty is to properly identify the corresponding limit. This part of the proof in [4] exploits in a crucial way the particular geometry of small inclusions as opposed to the large sheets of our work. (Note, however, that the main ideas in [4] could certainly be adapted to our setting). The proof 
that we develop in the present paper does not rely on this notion of two-scale convergence on surfaces but instead recovers directly the convergence of the currents to the appropriate term in the sense of distribution; cf. Proposition 4.4. This aspect of our work, and in particular the introduction of the function $\alpha^{d}(t)$, is close, in spirit at least, to the unfolding method developed in [12].

From a physical viewpoint, the plasmonic structure analyzed here has been proposed as a type of metamaterial that may achieve the epsilon-near-zero effect. According to this effect, a macroscopic electromagnetic wave can propagate through the structure almost without any phase delay. This possibility has been recently predicted for isotropic and homogeneous metallic sheets hosted by relatively simple, anisotropic dielectrics (ambient media) by use of classical solutions to Maxwell's equations via the Bloch wave theory [21, 23]. Our analysis here is more general since it relies on intrinsic properties of Maxwell's equations, without recourse to particular solutions. Thus, our homogenization result is a promising tool for understanding how the epsilon-near-zero effect can possibly emerge in a broad class of plasmonic structures. The implications of our homogenization outcome are the subject of work in progress.

To link our homogenization result to predictions related to the epsilon-nearzero effect, e.g., [21, 23], consider cell problem (5) in the simple case with $\nabla_{y}$. $\varepsilon(\boldsymbol{x}, \boldsymbol{y}) \equiv 0$. By this hypothesis, we deduce that the corrector field must vanish, i.e., $\boldsymbol{\chi}(\boldsymbol{x}, \boldsymbol{y}) \equiv 0$. Hence, formula (4) for $\varepsilon^{\mathrm{eff}}$ reduces to the average

$$
\varepsilon^{\mathrm{eff}}=\int_{Y} \varepsilon(\boldsymbol{y}) \mathrm{d} y-\frac{1}{i \omega} \int_{\Sigma}\left\{\sigma(\boldsymbol{y}) P_{T}\left(I_{n}\right)\right\} \mathrm{d} o_{y},
$$

under the additional, simplifying assumption that the dielectric permittivity, $\varepsilon^{d}$, of the ambient medium and the surface conductivity, $\sigma^{d}$, of each sheet depend only on the fast coordinate of the problem. For a plasmonic sheet such as doped graphene it is possible to have $\operatorname{Im} \sigma>0$ and $\operatorname{Im} \sigma \gg \operatorname{Re} \sigma>0$ [10, 13]. Thus, by inspection of (48) one observes that $\sigma=\sigma(\omega)=\sigma^{d}(\omega) / d$ can possibly be tuned so that at least one of the eigenvalues of $\varepsilon^{\text {eff }}$ is close to zero. This in turn implies that an electromagnetic wave propagating in the appropriate direction, determined by the respective eigenvector of $\varepsilon^{\text {eff }}$, may experience almost no phase delay. For examples in the relatively simple setting with a diagonal $\varepsilon$ and scalar constant $\sigma$, the reader is referred to [21, 23].

Specifically, if one chooses $\varepsilon(\boldsymbol{y})=\operatorname{diag}\left(\varepsilon_{x}(\boldsymbol{y}), \varepsilon_{y}(\boldsymbol{y}), \varepsilon_{z}(\boldsymbol{y})\right)$ with $\varepsilon_{x}=$ const., $\varepsilon_{y}(\boldsymbol{y})=\varepsilon_{z}(\boldsymbol{y})=\varepsilon_{z, 0} f\left(y_{1}\right), \varepsilon_{z, 0}=$ const. and $\sigma=$ const. for some positive and bounded function $f$ [21], by (48) the effective dielectric permittivity becomes

$$
\varepsilon^{\mathrm{eff}}=\operatorname{diag}\left(\varepsilon_{x}, \quad \varepsilon_{z, 0} \int_{0}^{1} f\left(y_{1}\right) \mathrm{d} y_{1}+i \sigma / \omega, \quad \varepsilon_{z, 0} \int_{0}^{1} f\left(y_{1}\right) \mathrm{d} y_{1}+i \sigma / \omega\right) .
$$

Notice that if $\operatorname{Re} \sigma \approx 0$ and $\operatorname{Im} \sigma>0$, the two diagonal elements of $\varepsilon^{\text {eff }}$ are close to zero if $\omega$ or $d$ is adjusted so that the following relation holds:

$$
d \approx d_{0}:=\frac{-i \sigma^{d}(\omega)}{\omega \varepsilon_{z, 0}}\left(\int_{0}^{1} f\left(y_{1}\right) \mathrm{d} y_{1}\right)^{-1}
$$


Note that the quantity $-i \sigma^{d} /\left(\omega \varepsilon_{z, 0}\right)$ is the plasmonic length, which expresses the scale for the decay of a surface plasmon-polariton away from the sheet in the case of transverse-magnetic polarization [23]. The condition $d \approx d_{0}$ has dramatic consequences in the dispersion of macroscopic waves through the plasmonic structure [21].

This discussion points to a few open problems with direct implications in plasmonics. For instance, it is of interest to define the epsilon-near-zero effect in situations where the dielectric permittivity of the ambient medium or the conductivity of the metallic sheet also depend on slow spatial variables (in isotropic or anisotropic settings). A related issue is to understand the role of the corrector field if $\nabla_{y} \cdot \varepsilon(\boldsymbol{x}, \boldsymbol{y}) \neq 0$. Our assumption that the ambient medium and sheet are non-magnetic can be deemed as restrictive, and could in principle be relaxed. In the presence of magnetic media, the homogenized Maxwell equations may include an effective magnetic permeability, $\mu^{\text {eff }}$, that should combine bulk and surface averages. In fact, the jump condition across the sheet can be generalized to also include a discontinuity in the tangential electric field which may be relevant to the magnetoelectric effect [36]. This and other generalizations can lead to rich homogenization problems in plasmonics.

\section{Appendix A. Two-scale convergence: A few results}

First, we recall the following classical definition and corresponding theorem [1].

Definition A.1. A sequence $\boldsymbol{u}^{d}$ in $L^{2}\left(\Omega ; \mathbb{C}^{3}\right)$ is said to two-scale converge to $\boldsymbol{u}^{(0)} \in L^{2}\left(\Omega \times Y ; \mathbb{C}^{3}\right)$ if

$$
\lim _{d \rightarrow 0} \int_{\Omega} \boldsymbol{u}^{d}(\boldsymbol{x}) \cdot \mathbf{\Psi}(\boldsymbol{x}, \boldsymbol{x} / d) \mathrm{d} x=\int_{\Omega} \int_{Y} \boldsymbol{u}^{(0)}(\boldsymbol{x}, \boldsymbol{y}) \cdot \Psi(\boldsymbol{x}, \boldsymbol{y}) \mathrm{d} y \mathrm{~d} x,
$$

for all test functions $\boldsymbol{\Psi} \in C_{0}\left(\Omega ; C_{\#}\left(Y ; \mathbb{C}^{3}\right)\right)$.

Theorem A.2. If the sequence $\boldsymbol{u}^{d}$ is bounded in $L^{2}\left(\Omega ; \mathbb{C}^{3}\right)$, then there exists a subsequence which two-scale converge to a function $u^{(0)}(\boldsymbol{x}, \boldsymbol{y})$. Furthermore, the sequence $\boldsymbol{u}^{d}$ weakly converges in $L^{2}\left(\Omega ; \mathbb{C}^{3}\right)$ to the function

$$
\bar{u}(\boldsymbol{x})=\int_{Y} u^{(0)}(\boldsymbol{x}, \boldsymbol{y}) d y .
$$

Next, we prove the following lemma which is also relevant to our exposition.

Lemma A.3. Let $\boldsymbol{f} \in L_{\#}^{2}\left(Y ; \mathbb{C}^{3}\right)$ be such that

$$
\nabla_{y} \times \boldsymbol{f}(\boldsymbol{x}, \boldsymbol{y})=0 \quad \text { in } \mathcal{D}^{\prime}(\Omega \times Y), \quad \int_{Y} \boldsymbol{f}(\boldsymbol{x}, \boldsymbol{y}) \mathrm{d} y=0 .
$$

Then, there exists a scalar function $\varphi(\boldsymbol{x}, \boldsymbol{y}) \in L^{2}\left(\Omega ; H_{\#}^{1}(Y)\right)$ such that

$$
f(\boldsymbol{x}, \boldsymbol{y})=\nabla_{y} \varphi(\boldsymbol{x}, \boldsymbol{y}) .
$$


This result is a slight variation of Lemma B.5 in [40]. We give the proof for the sake of completeness.

Proof. We can write the following Fourier expansion of $\boldsymbol{f}$ in $Y$ :

$$
\boldsymbol{f}(\boldsymbol{x}, \boldsymbol{y})=\sum_{\boldsymbol{k} \in \mathbb{Z}^{3}} \boldsymbol{c}_{\boldsymbol{k}}(\boldsymbol{x}) e^{i 2 \pi \boldsymbol{k} \cdot \boldsymbol{y}} .
$$

The conditions on $\boldsymbol{f}$ imply that $\boldsymbol{c}_{\boldsymbol{k}} \times \boldsymbol{k}=0$ for all $\boldsymbol{k} \in \mathbb{Z}^{3}, \boldsymbol{c}_{0}=0$. In particular, for $\boldsymbol{k} \neq 0$, the vector $\boldsymbol{c}_{\boldsymbol{k}}$ is parallel to $\boldsymbol{k}$; and if we define $d_{\boldsymbol{k}}=\frac{\boldsymbol{c}_{\boldsymbol{k}} \cdot \boldsymbol{k}}{i 2 \pi|\boldsymbol{k}|^{2}}$ then we have $\boldsymbol{c}_{\boldsymbol{k}}=(i 2 \pi) d_{l} \boldsymbol{k}$. This in turn implies that the function

$$
\varphi(\boldsymbol{x}, \boldsymbol{y})=\sum_{\boldsymbol{k} \in \mathbb{Z}^{3} \backslash\{0\}} d_{l} e^{i 2 \pi \boldsymbol{k} \cdot \boldsymbol{y}}
$$

satisfies

$$
\nabla_{y} \varphi(\boldsymbol{x}, \boldsymbol{y})=\sum_{\boldsymbol{k} \in \mathbb{Z}^{3} \backslash\{0\}} i 2 \pi d_{\boldsymbol{k}} \boldsymbol{k} e^{i 2 \pi \boldsymbol{k} \cdot \boldsymbol{y}}=\boldsymbol{f}(\boldsymbol{x}, \boldsymbol{y}) .
$$

Furthermore, $\varphi(\boldsymbol{x}, \boldsymbol{y}) \in L^{2}\left(\Omega ; H_{\#}^{1}(Y)\right)$ since $\boldsymbol{f} \in L_{\#}^{2}\left(Y ; \mathbb{C}^{3}\right)$.

\section{Appendix B. General hypersurfaces $\Sigma^{d}$}

In this section, we generalize the main result to non-flat hypersurfaces $\Sigma$. In particular, we show how to prove our main result (4) when the hypersurface $\Sigma$ is not necessarily a plane, but forms the graph of a smooth $Y^{\prime}$-periodic function $h$ (for which, for simplicity, we assume that $-1 \leq h \leq 1$ ). More precisely, we still assume that the domain $\Omega$ has the form

$$
\Omega=\Sigma^{\prime} \times \Gamma,
$$

where $\Sigma^{\prime}$ is a smooth bounded subset of $\mathbb{R}^{2}$ and $\Gamma=(-L, L)$. But we now take

$$
\Sigma^{d}=\cup_{k \in \Gamma^{d}}\left\{\left(\boldsymbol{x}^{\prime}, d h\left(\boldsymbol{x}^{\prime} / d\right)+k d\right) ; \boldsymbol{x}^{\prime} \in \Sigma^{\prime}\right\},
$$

where $\Gamma^{d}=\{k \in \mathbb{Z} ; k d \in(-L+d, L-d)\}$. Note that this definition of $\Gamma^{d}$ (and the assumption $-1 \leq h \leq 1$ ) ensures that $\Sigma^{d}$ does not intersect the boundaries $\Sigma^{\prime} \times\{-L\}$ and $\Sigma^{\prime} \times\{L\}$. Finally, we recall that $\Sigma_{0}$ denotes the graph of $h$ in $Y$ :

$$
\Sigma_{0}=\left\{\left(y^{\prime}, h(y)\right) ; y^{\prime} \in Y^{\prime}\right\} .
$$

The only part in the proof of Theorem 2.4 that utilized the particular structure of $\Sigma^{d}$ was in the proof of Proposition 4.4. We will thus show in the following that the result of Proposition 4.4 still holds in the more general framework described above. In order to state the corresponding result, we introduce the matrix $P\left(\boldsymbol{y}^{\prime}\right)$, which expresses the projection onto the tangent space of $\Sigma_{0}$ at the point $\left(\boldsymbol{y}^{\prime}, h\left(\boldsymbol{y}^{\prime}\right)\right)$. For $\boldsymbol{x} \in \Sigma^{d}$, we thus have

$$
\boldsymbol{E}_{T}^{d}(\boldsymbol{x})=P\left(\boldsymbol{x}^{\prime} / d\right) \boldsymbol{E}^{d}(\boldsymbol{x}) .
$$

Our goal is then to prove the following proposition. 
Proposition B.1. Assume that $h \in W_{\#}^{2, \infty}\left(Y^{\prime}\right)$ and recall that $\boldsymbol{E}^{d}$ is bounded in $X^{d}$ and two-scale converges to the function $\boldsymbol{E}^{(0)}(\boldsymbol{x}, \boldsymbol{y})$. Then, for all functions $\boldsymbol{F}(\boldsymbol{x}, \boldsymbol{y})$ defined in $\Omega \times Y$ that are periodic with respect to $y$ and admit $\boldsymbol{F}, \nabla_{x} \boldsymbol{F}, \nabla_{y} \boldsymbol{F} \in L^{\infty}(\Omega \times Y)$, we have

$$
\begin{aligned}
\lim _{d \rightarrow 0} d \int_{\Sigma^{d}} \boldsymbol{E}_{T}^{d}(\boldsymbol{x}) \cdot \boldsymbol{F}_{T}(\boldsymbol{x}, \boldsymbol{x} / d) \mathrm{d} o_{x}= \\
\qquad \int_{\Omega} \int_{\Sigma_{0}} P(\boldsymbol{y}) \boldsymbol{E}^{(0)}(\boldsymbol{x}, \boldsymbol{y}) \cdot P(\boldsymbol{y}) F(\boldsymbol{x}, \boldsymbol{y}) \mathrm{d} o_{y} \mathrm{~d} x .
\end{aligned}
$$

Proof. As in the proof of Proposition 4.4, the key step is the introduction of the following function (defined for $t \in(0,1)$ ):

$$
\begin{aligned}
\alpha^{d}(t)= & d \int_{\Sigma^{d}} \boldsymbol{E}_{T}^{d}\left(\boldsymbol{x}^{\prime}, x_{3}+t d\right) \cdot \boldsymbol{F}_{T}\left(\boldsymbol{x}^{\prime}, x_{3}+t d ; \boldsymbol{x}^{\prime} / d, x_{3} / d\right) \mathrm{d} o_{x} \\
= & \sum_{k \in \Gamma^{d}} d \int_{\Sigma^{\prime}} P\left(\frac{\boldsymbol{x}^{\prime}}{d}\right) \boldsymbol{E}^{d}\left(\boldsymbol{x}^{\prime},(k+t) d+d h\left(\boldsymbol{x}^{\prime} / d\right)\right) \\
& \quad P\left(\frac{\boldsymbol{x}^{\prime}}{d}\right) \boldsymbol{F}\left(\boldsymbol{x}^{\prime},(k+t) d+d h\left(\boldsymbol{x}^{\prime} / d\right) ; \boldsymbol{x}^{\prime} / d, h\left(\boldsymbol{x}^{\prime} / d\right)\right) \sqrt{1+\left|\nabla h\left(\frac{\boldsymbol{x}^{\prime}}{d}\right)\right|^{2}} d \boldsymbol{x}^{\prime} .
\end{aligned}
$$

The main difficulty is to derive the appropriate bounds on $\alpha^{d}$ and its derivative (see Lemma 4.6). For this purpose, we introduce the diffeomorphisms $\boldsymbol{g}: \mathbb{R}^{3} \rightarrow \mathbb{R}^{3}$ and $\boldsymbol{g}^{d}: \mathbb{R}^{3} \rightarrow \mathbb{R}^{3}$ defined by $\boldsymbol{g}(\boldsymbol{x}):=\left(\boldsymbol{x}^{\prime}, h\left(\boldsymbol{x}^{\prime}\right)+x_{3}\right)$, and $\boldsymbol{g}^{d}(\boldsymbol{x}):=d \boldsymbol{g}(\boldsymbol{x} / d)=\left(\boldsymbol{x}^{\prime}, d h\left(\boldsymbol{x}^{\prime} / d\right)+x_{3}\right)$. We have $\Sigma^{d}=\boldsymbol{g}^{d}\left(\widetilde{\Sigma}^{d}\right)$, where

$$
\widetilde{\Sigma}^{d}=\cup_{k \in \Gamma^{d}} \Sigma^{\prime} \times\{k d\},
$$

and $\Omega=\boldsymbol{g}^{d}\left(\widetilde{\Omega}^{d}\right)$, where

$$
\widetilde{\Omega}^{d}=\left\{\left(x^{\prime}, x_{3}\right) ; x^{\prime} \in \Sigma^{\prime},-L-d h\left(x^{\prime} / d\right) \leq x_{3} \leq L-d h\left(x^{\prime} / d\right)\right\}
$$

(note that $\left|\Omega \Delta \widetilde{\Omega}^{d}\right| \leq C d$ ). We also define

$$
\widetilde{\boldsymbol{E}}^{d}(\boldsymbol{x})=\boldsymbol{E}^{d}\left(\boldsymbol{g}^{d}(\boldsymbol{x})\right) \nabla \boldsymbol{g}^{d}(\boldsymbol{x}), \quad x \in \widetilde{\Omega}^{d}
$$

that is, $\widetilde{\boldsymbol{E}}_{i}^{d}(\boldsymbol{x})=\sum_{j=1}^{3} \boldsymbol{E}_{j}\left(\boldsymbol{g}^{d}(\boldsymbol{x})\right) \partial_{i} \boldsymbol{g}_{j}^{d}(\boldsymbol{x})$. This is a natural definition when $\boldsymbol{E}^{d}$ is the gradient of a potential (that is when $\boldsymbol{E}^{d}$ is curl free). We will see below that this change of function also preserves the curl estimates that played a crucial role in the proof of Lemma 4.6. More precisely, we will make use of the following properties:

1. Since $\nabla \boldsymbol{g}^{d}(\boldsymbol{x})=\nabla \boldsymbol{g}(\boldsymbol{x} / d)$, we have $\left\|\partial_{i} \boldsymbol{g}_{j}^{d}\right\|_{L^{\infty}} \leq C$ for all $i, j$, independently of $d$. Furthermore, a simple computation gives

$$
\left|\operatorname{det} \nabla \boldsymbol{g}^{d}(\boldsymbol{x})\right|=1 \text {. }
$$

In particular, we have (with $d$-independent constants)

$$
\int_{\widetilde{\Omega}^{d}}\left|\widetilde{\boldsymbol{E}}^{d}(\boldsymbol{x})\right|^{2} d \boldsymbol{x} \leq C \int_{\Omega}\left|\boldsymbol{E}^{d}(\boldsymbol{x})\right|^{2} d \boldsymbol{x} \leq C .
$$


2. For $\boldsymbol{x} \in \widetilde{\Sigma}^{d}$, the projection $\widetilde{\boldsymbol{E}}_{T}^{d}$ onto the tangent plane to $\widetilde{\Sigma}^{d}$ only depends on $\boldsymbol{E}_{T}^{d}$, the projection of $\boldsymbol{E}^{d}$ onto the tangent plane to $\Sigma^{d}$. Indeed we can write

$$
\begin{aligned}
\widetilde{\boldsymbol{E}}_{T}^{d}(\boldsymbol{x}) & =\left(\widetilde{\boldsymbol{E}}_{1}^{d}(\boldsymbol{x}), \widetilde{\boldsymbol{E}}_{2}^{d}(\boldsymbol{x}), 0\right)^{T} \\
& =\left(\partial_{1} \boldsymbol{g}^{d}(\boldsymbol{x}) \cdot \boldsymbol{E}^{d}\left(\boldsymbol{g}^{d}(\boldsymbol{x})\right), \partial_{2} g^{d}(\boldsymbol{x}) \cdot \boldsymbol{E}^{d}\left(\boldsymbol{g}^{d}(\boldsymbol{x})\right), 0\right)^{T} \\
& =\left(\partial_{1} \boldsymbol{g}^{d}(\boldsymbol{x}) \cdot \boldsymbol{E}_{T}^{d}\left(\boldsymbol{g}^{d}(\boldsymbol{x})\right), \partial_{2} \boldsymbol{g}^{d}(\boldsymbol{x}) \cdot \boldsymbol{E}_{T}^{d}\left(\boldsymbol{g}^{d}(\boldsymbol{x})\right), 0\right)^{T} .
\end{aligned}
$$

In the last equality we used the fact that $\partial_{1} g^{d}$ and $\partial_{2} g^{d}$ are tangent vectors to $\Sigma^{d}$. Using the fact that the vector $\left(-\partial_{1} h,-\partial_{2} h, 1\right)$ is normal to $\Sigma_{d}$ (it is the vector $\partial_{1} g^{d} \times \partial_{2} g^{d}$ ), we can rewrite this equality as

$$
\widetilde{\boldsymbol{E}}_{T}^{d}(\boldsymbol{x})=M\left(\boldsymbol{x}^{\prime} / d\right) \boldsymbol{E}_{T}^{d}\left(\boldsymbol{g}^{d}(\boldsymbol{x})\right),
$$

with the matrix

$$
M\left(\boldsymbol{x}^{\prime}\right)=\left(\begin{array}{ccc}
1 & 0 & \partial_{1} h\left(\boldsymbol{x}^{\prime}\right) \\
0 & 1 & \partial_{2} h\left(\boldsymbol{x}^{\prime}\right) \\
-\partial_{1} h\left(\boldsymbol{x}^{\prime}\right) & -\partial_{2} h\left(\boldsymbol{x}^{\prime}\right) & 1
\end{array}\right) .
$$

This $M\left(\boldsymbol{x}^{\prime}\right)$ is smooth and invertible. (The latter attribute can be readily deduced from the determinant of $M\left(\boldsymbol{x}^{\prime}\right)$, which is $1+\left|\partial_{1} h\left(\boldsymbol{x}^{\prime} / d\right)\right|^{2}+$ $\left.\left|\partial_{2} h\left(\boldsymbol{x}^{\prime} / d\right)\right|^{2}\right)$. We can also write

$$
\widetilde{\boldsymbol{E}}_{T}^{d}(\boldsymbol{x})=M\left(\boldsymbol{x}^{\prime} / d\right) P\left(\boldsymbol{x}^{\prime} / d\right) \boldsymbol{E}^{d}\left(\boldsymbol{g}^{d}(\boldsymbol{x})\right) .
$$

3. The definition of $g$ immediately gives $\widetilde{\boldsymbol{E}}_{3}^{d}(\boldsymbol{x})=\boldsymbol{E}_{3}^{d}\left(\boldsymbol{g}^{d}(\boldsymbol{x})\right)$ in $\widetilde{\Omega}^{d}$. Furthermore, since $\boldsymbol{E}_{3}^{d}$ is part of $\boldsymbol{E}_{T}^{d}$ on $\partial \widetilde{\Sigma} \times \Gamma$, using (B.3) we conclude that

$$
\int_{\Gamma^{d}} \int_{\Sigma^{\prime}}\left|\widetilde{\boldsymbol{E}}_{3}^{d}\left(\boldsymbol{x}^{\prime}, x_{3}\right)\right|^{2} d \boldsymbol{x}^{\prime} \mathrm{d} x_{3} \leq \int_{\Gamma^{d}} \int_{\Sigma}\left|\boldsymbol{E}_{3}^{d}\left(\boldsymbol{x}^{\prime}, x_{3}\right)\right|^{2} d \boldsymbol{x}^{\prime} \mathrm{d} x_{3} \leq C .
$$

4. The curl of $\widetilde{\boldsymbol{E}}^{d}$ only depends on the components of $\nabla \times \boldsymbol{E}^{d}$, and is thus bounded in $L^{2}$. Indeed, writing $\widetilde{\boldsymbol{E}}^{d}(\boldsymbol{x})=\sum_{j=1}^{3} E_{j}^{d}\left(\boldsymbol{g}^{d}(\boldsymbol{x})\right) \nabla \boldsymbol{g}_{j}^{d}(\boldsymbol{x})$, we find

$$
\begin{aligned}
\nabla \times \widetilde{\boldsymbol{E}}^{d}(\boldsymbol{x}) & =\sum_{j=1}^{3} \nabla\left(\boldsymbol{E}_{j}^{d}\left(\boldsymbol{g}^{d}(x)\right) \times \nabla \boldsymbol{g}_{j}^{d}(\boldsymbol{x})\right. \\
& =\sum_{j, l=1}^{3} \partial_{l} \boldsymbol{E}_{j}^{d}\left(\boldsymbol{g}^{d}(x)\right) \nabla \boldsymbol{g}_{l}^{d}(x) \times \nabla \boldsymbol{g}_{j}^{d}(\boldsymbol{x}),
\end{aligned}
$$

where we used the chain rule $\nabla\left(\boldsymbol{E}_{j}(\boldsymbol{g}(\boldsymbol{x}))=\sum_{l=1}^{3} \partial_{l} \boldsymbol{E}_{j}(\boldsymbol{g}(\boldsymbol{x})) \nabla \boldsymbol{g}_{l}(\boldsymbol{x})\right.$. Using the anti-symmetry of the cross product, we deduce the relation

$$
\nabla \times \widetilde{\boldsymbol{E}}^{d}(\boldsymbol{x})=\frac{1}{2} \sum_{j \neq l} \nabla \boldsymbol{g}_{l}^{d}(\boldsymbol{x}) \times \nabla \boldsymbol{g}_{j}^{d}(\boldsymbol{x})\left[\partial_{l} \boldsymbol{E}_{j}^{d}\left(\boldsymbol{g}^{d}(\boldsymbol{x})\right)-\partial_{j} \boldsymbol{E}_{l}^{d}\left(\boldsymbol{g}^{d}(\boldsymbol{x})\right)\right] .
$$


We thus see that $\nabla \times \widetilde{\boldsymbol{E}}^{d}(\boldsymbol{x})$ depends in a linear fashion on the components of $\nabla \times \boldsymbol{E}^{d}\left(\boldsymbol{g}^{d}(\boldsymbol{x})\right)$ and, since $\left\|\partial_{i} \boldsymbol{g}_{j}^{d}\right\|_{L^{\infty}} \leq C$, we obtain

$$
\left|\nabla \times \widetilde{\boldsymbol{E}}^{d}(\boldsymbol{x})\right|^{2} \leq C\left|\nabla \times \boldsymbol{E}^{d}\left(\boldsymbol{g}^{d}(\boldsymbol{x})\right)\right|^{2} .
$$

In particular, (B.3) implies the estimates

$$
\int_{\widetilde{\Omega}^{d}}\left|\nabla \times \widetilde{\boldsymbol{E}}^{d}(\boldsymbol{x})\right|^{2} d \boldsymbol{x} \leq C \int_{\Omega}\left|\nabla \times \boldsymbol{E}^{d}(\boldsymbol{x})\right|^{2} d \boldsymbol{x} \leq C .
$$

We are now ready to prove Proposition B.1. Using the change of variable introduced above, we can rewrite the function $\alpha^{d}(t)$ in a form similar to the one appearing in Proposition 4.4. Indeed, using (B.2) and (B.5), we find that

$$
\alpha^{d}(t)=\sum_{k \in \Gamma^{d}} d \int_{\Sigma^{\prime}} \widetilde{\boldsymbol{E}}_{T}^{d}\left(\boldsymbol{x}^{\prime},(k+t) d\right) \cdot \widetilde{\boldsymbol{F}}_{T}^{d}\left(\boldsymbol{x}^{\prime},(k+t) d ; \boldsymbol{x}^{\prime} / d, 0\right) \mathrm{d} x^{\prime}
$$

where

$$
\begin{aligned}
\widetilde{\boldsymbol{F}}_{T}^{d}(\boldsymbol{x}, \boldsymbol{y}) & =\left(M\left(\boldsymbol{y}^{\prime}\right)^{-1}\right)^{T} \boldsymbol{F}_{T}\left(\boldsymbol{g}^{d}(\boldsymbol{x}), \boldsymbol{g}(\boldsymbol{y})\right) \sqrt{1+\left|\nabla h\left(\boldsymbol{y}^{\prime}\right)\right|^{2}} \\
& =\left(M\left(\boldsymbol{y}^{\prime}\right)^{-1}\right)^{T} \boldsymbol{F}_{T}\left(\boldsymbol{x}^{\prime}, d h\left(\boldsymbol{y}^{\prime}\right)+x_{3}, \boldsymbol{g}(\boldsymbol{y})\right) \sqrt{1+\left|\nabla h\left(\boldsymbol{y}^{\prime}\right)\right|^{2}} .
\end{aligned}
$$

In order to finalize this step, we only need to show that the results of Lemma 4.5 and Lemma 4.6 hold in our framework. The proof of Lemma 4.6 requires only appropriate bounds on $\widetilde{\boldsymbol{E}}^{d}$ and $\widetilde{\boldsymbol{F}}^{d}$. In particular, we realize that $\widetilde{\boldsymbol{F}}^{d}$ satisfies

$$
\left\|\widetilde{\boldsymbol{F}}^{d}\right\|_{L^{\infty}(\Omega \times Y)} \leq C, \quad\left\|\nabla_{x} \widetilde{\boldsymbol{F}}^{d}\right\|_{L^{\infty}(\Omega \times Y)} \leq C, \quad\left\|\nabla_{y} \widetilde{\boldsymbol{F}}^{d}\right\|_{L^{\infty}(\Omega \times Y)} \leq C,
$$

(with constant $C$ independent of $d$ ) which, together with the bounds (B.4), (B.7) and (B.8) are all that we need to prove Lemma 4.6. These same bounds are also sufficient to show that $\alpha^{d}(t)$ is bounded in $L^{2}(0,1)$. To prove Lemma 4.5. we therefore only need to identify the limit of $\int_{0}^{1} \alpha^{d}(t) \varphi(t) \mathrm{d} t$. Using (B.6) and $(\underline{B .2})$, we write

$$
\begin{array}{r}
\int_{0}^{1} \alpha^{d}(t) \varphi(t) \mathrm{d} t=\int_{\Sigma \times \tilde{\Gamma}^{d}} M\left(\frac{\boldsymbol{x}^{\prime}}{d}\right) P\left(\frac{\boldsymbol{x}^{\prime}}{d}\right) \boldsymbol{E}^{d}\left(\boldsymbol{x}^{\prime}, d h\left(\frac{\boldsymbol{x}^{\prime}}{d}\right)+x_{3}\right) \\
\cdot\left(M\left(\frac{\boldsymbol{x}^{\prime}}{d}\right)^{-1}\right)^{T} \boldsymbol{F}_{T}\left(\boldsymbol{x}^{\prime}, d h\left(\frac{\boldsymbol{x}^{\prime}}{d}\right)+x_{3}, \boldsymbol{g}\left(\frac{\boldsymbol{x}^{\prime}}{d}, 0\right)\right) \varphi\left(x_{3} / d\right) \\
=\int_{\Omega^{\prime}} P\left(\frac{\boldsymbol{x}^{\prime}}{d}\right) \boldsymbol{E}^{d}\left(\boldsymbol{x}^{\prime}, x_{3}\right) \cdot P\left(\frac{\boldsymbol{x}^{\prime}}{d}\right) \boldsymbol{F}\left(\boldsymbol{x}^{\prime}, x_{3}, \boldsymbol{g}\left(\frac{\boldsymbol{x}^{\prime}}{d}, 0\right)\right) \\
\varphi\left(\frac{x_{3}^{\prime}}{d}-h\left(\frac{\boldsymbol{x}^{\prime}}{d}\right)\right) \sqrt{1+\left|\nabla h\left(\frac{\boldsymbol{x}^{\prime}}{d}\right)\right|^{2}} d \boldsymbol{x}
\end{array}
$$


with $\tilde{\Gamma}^{d}=\cup_{k \in \Gamma^{d}}[k d,(k+1) d]=\left[d k_{0}, d k_{1}\right]$ and $\Omega^{\prime}=\left\{\left(\boldsymbol{x}^{\prime}, x_{3}\right) ; \boldsymbol{x}^{\prime} \in \Sigma^{\prime}, d k_{0}+\right.$ $\left.d h\left(\boldsymbol{x}^{\prime} / d\right)<x_{3}<d k_{1}+d h\left(\boldsymbol{x}^{\prime} / d\right)\right\}$. The usual properties of two-scale convergence imply that the above expression converges to

$$
\begin{gathered}
\int_{\Omega} \int_{Y} P\left(\boldsymbol{y}^{\prime}\right) \boldsymbol{E}^{(0)}(\boldsymbol{x}, \boldsymbol{y}) \cdot P\left(\boldsymbol{y}^{\prime}\right) \boldsymbol{F}\left(\boldsymbol{x}, \boldsymbol{g}\left(\boldsymbol{y}^{\prime}, 0\right)\right) \varphi\left(y_{3}-h\left(\boldsymbol{y}^{\prime}\right)\right) \sqrt{1+\left|\nabla h\left(\boldsymbol{y}^{\prime}\right)\right|^{2}} d \boldsymbol{y} d \boldsymbol{x} \\
=\int_{\Omega} \int_{Y} P\left(\boldsymbol{y}^{\prime}\right) \boldsymbol{E}^{(0)}(\boldsymbol{x}, \boldsymbol{y}) \cdot P\left(\boldsymbol{y}^{\prime}\right) \boldsymbol{F}\left(\boldsymbol{x}, \boldsymbol{y}^{\prime}, h\left(\boldsymbol{y}^{\prime}\right)\right) \\
\varphi\left(y_{3}-h\left(\boldsymbol{y}^{\prime}\right)\right) \sqrt{1+\left|\nabla h\left(\boldsymbol{y}^{\prime}\right)\right|^{2}} d \boldsymbol{y} d \boldsymbol{x} \\
=\int_{\Omega} \int_{Y} P\left(\boldsymbol{y}^{\prime}\right) \boldsymbol{E}^{(0)}\left(\boldsymbol{x}, \boldsymbol{y}^{\prime}, y_{3}+h\left(\boldsymbol{y}^{\prime}\right)\right) \cdot P\left(\boldsymbol{y}^{\prime}\right) \boldsymbol{F}\left(\boldsymbol{x}, \boldsymbol{y}^{\prime}, h\left(\boldsymbol{y}^{\prime}\right)\right) \\
\varphi\left(y_{3}\right) \sqrt{1+\left|\nabla h\left(\boldsymbol{y}^{\prime}\right)\right|^{2}} d \boldsymbol{y} d \boldsymbol{x} .
\end{gathered}
$$

It follows that $\alpha^{d}(t)$ converges $L^{2}(0,1)$ weakly to

$$
\begin{aligned}
\alpha^{0}(t)=\int_{\Omega} \int_{Y} P\left(\boldsymbol{y}^{\prime}\right) \boldsymbol{E}^{(0)}\left(\boldsymbol{x}, \boldsymbol{y}^{\prime}, t+h\left(\boldsymbol{y}^{\prime}\right)\right) \cdot P\left(\boldsymbol{y}^{\prime}\right) \boldsymbol{F}\left(\boldsymbol{x}, \boldsymbol{y}^{\prime}, h\left(\boldsymbol{y}^{\prime}\right)\right) \\
\times \sqrt{1+\left|\nabla h\left(\boldsymbol{y}^{\prime}\right)\right|^{2}} d \boldsymbol{y} d \boldsymbol{x} .
\end{aligned}
$$

Analogously to the proof of Proposition 4.4, we can thus conclude that

$$
\begin{aligned}
& \lim _{d \rightarrow 0} d \int_{\Sigma^{d}} \boldsymbol{E}_{T}^{d}(\boldsymbol{x}) \cdot \boldsymbol{F}_{T}(\boldsymbol{x}, \boldsymbol{x} / d) \mathrm{d} o_{x} \\
& =\alpha^{0}(0) \\
& =\int_{\Omega} \int_{Y^{\prime}} P\left(\boldsymbol{y}^{\prime}\right) \boldsymbol{E}^{(0)}\left(\boldsymbol{x}, \boldsymbol{y}^{\prime}, h\left(\boldsymbol{y}^{\prime}\right)\right) \cdot P\left(\boldsymbol{y}^{\prime}\right) \boldsymbol{F}\left(\boldsymbol{x}, \boldsymbol{y}^{\prime}, h\left(\boldsymbol{y}^{\prime}\right)\right) \sqrt{1+\left|\nabla h\left(\boldsymbol{y}^{\prime}\right)\right|^{2}} d \boldsymbol{y}^{\prime} d \boldsymbol{x} \\
& =\int_{\Omega} \int_{\Sigma_{0}} P(\boldsymbol{y}) \boldsymbol{E}^{(0)}(\boldsymbol{x}, \boldsymbol{y}) \cdot P(\boldsymbol{y}) \boldsymbol{F}(\boldsymbol{x}, \boldsymbol{y}) \mathrm{d} o_{y} d \boldsymbol{x} .
\end{aligned}
$$

This completes the proof of Proposition B.1.

\section{Acknowledgments}

We wish to thank Professors Mitchell Luskin and Robert V. Kohn for useful discussions. The first two authors (MM and DM) have been supported by ARO MURI via Award No. W911NF-14-1-0247. The authors acknowledge partial support by NSF through Awards DMS-1912847 (MM), DMS-1412769 (DM), and DMS-1501067 (AM).

\section{References}

[1] G. Allaire. Homogenization and two-scale convergence. SIAM Journal on Mathematical Analysis, 23(6):1482-1518, 1992. 
[2] G. Allaire, A. Damlamian, and U. Hornung. Two-scale convergence on periodic surfaces and applications. In A. Bourgeat, C. Carasso, S. Luckhaus, and A. Mikelic, editors, Proceedings of the Conference: Mathematical Modelling of Flow Through Porous Media, pages 15-25, 1995.

[3] Y. Amirat and V. V. Shelukhin. Homogenization of the time harmonic Maxwell equations and the frequency dispersion effect. Journal de Mathématiques Pures et Appliquées, 95(4):420-443, 2011.

[4] Y. Amirat and V. V. Shelukhin. Homogenization of time harmonic Maxwell equations: the effect of interfacial currents. Mathematical Methods in the Applied Sciences, 40(8):3140-3162, 2017.

[5] M. Artola. Homogenization and electromagnetic wave propagation in composite media with high conductivity inclusions. In G. D. Maso and G. Dell'Antonio, editors, Proceedings of the Second Workshop on Composite Media and Homogenization Theory, pages 1-15, 1995.

[6] A. Bensoussan, J.-L. Lions, and G. Papanicolaou. Asymptotic Analysis for Periodic Structures, volume 5 of Studies in Mathematics and its Applications. North-Holland, $1^{\text {st }}$ edition, 1978.

[7] Y. V. Bludov, A. Ferreira, N. Peres, and M. I. Vasileskiy. A primer on surface plasmon-polaritons in graphene. International Journal of Modern Physics, 27(10):1341001, 2013.

[8] J. D. Caldwell, I. Vurgaftman, J. G. Tischler, O. J. Glembocki, J. C. Owrutsky, and T. L. Reinecke. Atomic-scale photonic hybrids for mid-infrared and terahertz nanophotonics. Nature Nanotechnology, 11(27):9-15, 2016.

[9] L. Cao, Y. Zhang, W. Allegretto, and Y. Lin. Multiscale asymptotic method for Maxwell's equations in composite materials. SIAM Journal on Numerical Analysis, 47(6):4257-4289, 2010.

[10] J. Cheng, W. L. Wang, H. Mosallaei, and E. Kaxiras. Surface plasmon engineering in graphene functionalized with organic molecules: A multiscale theoretical investigation. Nano Letters, 14:50-56, 2014.

[11] D. Cioranescu and P. Donato. An Introduction to Homogenization, volume 17 of Oxford Lecture Series in Mathematics and its Applications. Oxford University Press, $1^{\text {st }}$ edition, 1999.

[12] D. Cioranescu, P. Donato, and R. Zaki. The periodic unfolding method in perforated domains. Port. Math. (N.S.), 63(4):467-496, 2006. ISSN $0032-5155$.

[13] A. N. Grigorenko, M. Polini, and K. S. Novoselov. Graphene plasmonics. Nature Photonics, 6(11):749-758, 2012. 
[14] S. Guenneau, F. Zolla, and A. Nicolet. Homogenization of 3d finite photonic crystals with heterogeneous permittivity and permeability. Waves in Random and Complex Media, 17(4):653-697, 2007.

[15] P. Henning, M. Ohlberger, and B. Verfürth. A new heterogeneous multiscale method for time-harmonic Maxwell's equations. SIAM Journal on Numerical Analysis, 54(6):3493-3522, 2017.

[16] S. Jahani and Z. Jacob. All-dielectric metamaterials. Nature Nanotechnology, 11:23-36, 2016.

[17] G. Kristensson. Homogenization of corrugated interfaces in electromagnetics. Progress in Electromagnetics Research, 55:1-31, 2005.

[18] Y. Li, S. Kita, P. Munoz, O. Reshef, D. I. Vulis, M. Yin, M. Lončar, and E. Mazur. On-chip zero-index metamaterials. Nature Photonics, 9:738-743, 2015 .

[19] T. Low, A. Chaves, J. D. Caldwell, A. Kumar, N. X. Fang, P. Avouris, T. F. Heinz, F. Guinea, L. Martin-Moreno, and F. Koppens. Polaritons in layered two-dimensional materials. Nature Materials, 16:182-193, 2017.

[20] M. Maier, D. Margetis, and M. Luskin. Dipole excitation of surface plasmon on a conducting sheet: finite element approximation and validation. Journal of Computational Physics, 339:126-145, 2017.

[21] M. Maier, M. Mattheakis, E. Kaxiras, M. Luskin, and D. Margetis. Universal behavior of dispersive Dirac cone in gradient-index plasmonic metamaterials. Physical Review B, 97(3):035307/1-7, 2018.

[22] M. Maier, M. Mattheakis, E. Kaxiras, M. Luskin, and D. Margetis. Homogenization of plasmonic crystals: Seeking the epsilon-near-zero effect. Proceedings of the Royal Society A: Mathematical, Physical, and Engineering Sciences, 475, 2019. doi: 10.1098/rspa.2019.0220. URL https://arxiv.org/abs/1809.08276.

[23] M. Mattheakis, C. A. Valagiannopoulos, and E. Kaxiras. Epsilon-nearzero behavior from plasmonic Dirac point: Theory and realization using two-dimensional materials. Physical Review B, 94:201404(R), 2016.

[24] P. Moitra, Y. Yang, Z. Anderson, I. I. Kravchenko, D. P. Briggs, and J. Valentine. Realization of an all-dielectric zero-index optical metamaterial. Nature Photonics, 7:791-795, 2013.

[25] P. Monk. Finite Element Methods for Maxwell's Equations. Numerical Mathematics and Scientific Computation. Oxford University Press, 2003.

[26] C. Müller. Foundations of the Mathematical Theory of Electromagnetic Waves. Springer-Verlag, New York, 1969. 
[27] M. Neuss-Radu. Some extensions of two-scale convergence. C. R. Acad. Sci. Paris Sér. I Math., 322(9):899-904, 1996. ISSN 0764-4442.

[28] J. Nevard and J. B. Keller. Homogenization of rough boundaries and interfaces. SIAM Journal of Applied Mathematics, 57:1660-1686, 1997.

[29] G. Nguetseng. A general convergence result for a functional related to the theory of homogenization. SIAM Journal on Mathematical Analysis, 20(3): 608-623, 1989.

[30] G. Pavliotis and A. Stuart. Multiscale Methods: Averaging and Homogenization, volume 53 of Texts in Applied Mathematics. Springer, $1^{\text {st }}$ edition, 2008.

[31] J. M. Pitarke, V. M. Silkin, E. V. Chulkov, and P. M. Echenique. Theory of surface plasmons and surface-plasmon polaritons. Reports on Progress in Physics, 70:1-87, 2007.

[32] E. Sanchez-Palencia. Non-Homogeneous Media and Vibration Theory, volume 127 of Lecture Notes in Physics. Springer, $1^{\text {st }}$ edition, 1980.

[33] M. Schwartz. Principles of Electrodynamics. International Series in Pure and Applied Physics. McGraw-Hill Book Company, New York, 1972.

[34] M. Silveirinha and N. Engheta. Tunneling of electromagnetic energy through subwavelength channels and bends using $\epsilon$-near-zero materials. Physical Review Letters, 97:157403/1-4, 2006.

[35] D. Sjöberg, C. Engström, G. Kristensson, D. J. N. Wall, and N. Wellander. A Floquet-Bloch decomposition of Maxwell's equations applied to homogenization. SIAM Multiscale Modeling and Simulation, 4(1):149-171, 2005.

[36] U. Zülicke and R. Winkler. Magnetoelectric effect in bilayer graphene controlled by valley-isospin density. Physical Review B, 90:125412/1-5, 2014.

[37] B. Wang, X. Zhang, F. J. Garcia-Vidal, X. Yuan, and J. Teng. Strong coupling of surface plasmon polaritons in monolayer graphene sheet arrays. Physical Review Letters, 109:073901/1-5, 2012.

[38] N. Wellander. Homogenization of the Maxwell equations: Case i. linear theory. Applications of Mathematics, 46(1):29-51, 2001.

[39] N. Wellander. Homogenization of the Maxwell equations: Case ii. nonlinear conductivity. Applications of Mathematics, 47(3):255-283, 2002.

[40] N. Wellander and G. Kristensson. Homogenization of the Maxwell equations at fixed frequency. SIAM Journal on Applied Mathematics, 64(1): 170-195, 2003.

[41] N. I. Zheludev and E. Plum. Reconfigurable nanomechanical photonic metamaterials. Nature Nanotechnology, 11:16-22, 2016. 